\title{
Formation of contact in massive close binaries
}

\author{
S. Wellstein ${ }^{1}$, N. Langer ${ }^{1,2}$, and H. Braun ${ }^{3}$ \\ 1 Institut für Physik, Universität Potsdam, 14415 Potsdam, Germany \\ 2 Astronomical Institute, Utrecht University, Princetonplein 5, 3584 CC, Utrecht, The Netherlands \\ 3 Max-Planck-Institut für Astrophysik, 85740 Garching, Germany
}

Received 7 September 2000 / Accepted 23 January 2001

\begin{abstract}
We present evolutionary calculations for 74 close binaries systems with initial primary masses in the range $12 \ldots 25 M_{\odot}$, and initial secondary masses between 6 and $24 M_{\odot}$. The initial periods were chosen such that mass overflow starts during the core hydrogen burning phase of the primary (Case A), or shortly thereafter (Case B). We use a newly developed binary code with up-to-date physics input. Of particular relevance is the use of OPAL opacities, and the time-dependent treatment of semiconvective and thermohaline mixing. We assume conservative evolution for contact-free systems, i.e., no mass or angular momentum loss from those system except due to stellar winds. We investigate the borderline between contact-free evolution and contact, as a function of the initial system parameters. The fraction of the parameter space where binaries may evolve while avoiding contact - which we found already small for the least massive systems considered - becomes even smaller for larger initial primary masses. At the upper end of the considered mass range, no contact-free Case B systems exist. While for primary masses of $16 M_{\odot}$ and higher the Case A systems dominate the contact-free range, at primary masses of $12 M_{\odot}$ contact-free systems are more frequent for Case B. We identify the drop of the exponent $x$ in the main sequence mass-luminosity relation of the form $L \propto M^{x}$ as the main cause for this behaviour. For systems which evolve into contact, we find that this can occur for distinctively different reasons. While Case A systems are prone to contact due to reverse mass transfer during or after the primary's main sequence phase, all systems obtain contact for initial mass ratios below $\sim 0.65$, with a merger as the likely outcome. We also investigate the effect of the treatment of convection, and found it relevant for contact and supernova order in Case A systems, particularly for the highest considered masses. For Case B systems we find contact for initial periods above $\sim 10$ d. However, in that case (and for not too large periods) contact occurs only after the mass ratio has been reversed, due to the increased fraction of the donor's convective envelope. As most of the mass transfer occurs conservatively before contact is established, this delayed contact is estimated to yield to the ejection of only a fraction of the donor star's envelope. Our models yield the value of $\beta$, i.e., the fraction of the primaries envelope which is accreted by the secondary. We derive the observable properties of our systems after the major mass transfer event, where the mass gainer is a main sequence or supergiant $\mathrm{O}$ or early $\mathrm{B}$ type star, and the mass loser is a helium star. We point out that the assumption of conservative evolution for contact-free systems could be tested by finding helium star companions to O stars. Those are also predicted by non-conservative models, but with different periods and mass ratios. We describe strategies for increasing the probability to find helium star companions in observational search programs.
\end{abstract}

Key words. stars: evolution - stars: binaries - stars: blue stragglers - stars: circumstellar matter

\section{Introduction}

To understand the evolution of massive close binary system is relevant for many current astrophysical problems. Massive binaries provide the unique means to obtain accurate fundamental stellar properties of massive stars for various evolutionary stages. I.e., they have been used to derive masses of Wolf-Rayet stars (van der Hucht 2000)

Send offprint requests to: N. Langer,

e-mail: N.Langer@astro.uu.nl and to establish mass-luminosity relations for massive stars (Martin et al. 1998; Ostrov et al. 2000). They also lead to the existence of stellar configurations which do not evolve from single stars and which have highly exciting observational manifestations. Examples are the peculiar supernova 1987A, which several authors attribute to binary evolution (Podsiadlowski 1992; de Loore \& Vanbeveren 1992; Braun \& Langer 1995), short-period Wolf-Rayet binaries (e.g., Harries \& Hilditch 1997), massive X-ray binaries (Chevalier \& Ilovaisky 1998), supernovae of types Ib 
and Ic (Podsiadlowski et al. 1992), and $\gamma$-ray burster (Fryer et al. 1999)

However, the evolutionary theory of massive close binaries still suffers from fundamental uncertainties. The evolution of such systems has been modelled by various authors, e.g., Paczyński (1967), Kippenhahn (1969), de Loore \& De Greve (1992), Pols (1994), Vanbeveren et al. (1998a,b,c), Wellstein \& Langer (1999). One longstanding important question in binary models which include mass transfer is: how much of the transfered matter can actually be accreted by the secondary star? In most calculations so far, the fraction $\beta$ of the accreted matter which is retained on the accreting star is chosen as a constant, with $\beta=0.5$ being a frequent choice (e.g., Moers \& van den Heuvel 1989; De Greve \& De Loore 1992; De Loore \& De Greve 1992; Vanbeveren et al. 1998a,b,c). This means that half of the matter lost by the donor star is accreted by the mass gainer; the other half supposed to leave the binary system due to an undefined force, and carrying an amount of angular momentum which needs to be described by a second parameter (cf. Huang \& Taam 1990; Podsiadlowski et al. 1992). Also the assumption of a fully conservative mass transfer where all matter is accreted by the secondary, i.e. $\beta=1$, has been used. Physical models which yield the parameter $\beta$ as a function of the system parameters, or even as a function of time for a give system, are lacking.

Arguments of $\beta \simeq 0.5$ on average are being used in the literature (De Greve \& de Loore 1992; de Loore \& De Greve 1992; Vanbeveren et al. 1998a,b,c), and correspondingly massive close binary models using $\beta=0.5$ have been computed (see above). Here, we pursue a different approach. We adopt $\beta=1$ as long as the two stars in the binary system do not evolve into contact. We want to find out how many massive binaries actually avoid contact when $\beta=1$ is used, and whether the result can be compatible with an average value of $\beta \simeq 0.5$.

Mass transfer in systems with initial mass ratios very different from unity is known to lead to the rapid expansion of the accreting star and thus to a contact system (Benson 1970; Ulrich \& Burger 1976). Also systems with large initial periods are prone to develop contact, as convective envelopes of the donor stars lead to very high mass transfer rates (cf. Podsiadlowski et al. 1992). Pols (1994) found that Case $\mathrm{A}^{1}$ systems with primary masses in the range $8 \ldots 16 M_{\odot}$ avoid contact for initial mass ratios $q \gtrsim 0.7$ and initial periods $P_{0}>1.6 \mathrm{~d}$. However, Pols used opacities from Cox \& Stewart (1970), the Schwarzschild criterion for convection, and instantaneous thermohaline mixing. In the present work, we reconsider the work done by Pols using updated physics input, and we extend the considered parameters space towards higher masses and larger initial periods.

\footnotetext{
${ }^{1}$ Case A: mass transfer during central hydrogen burning; Case B: mass transfer after the end of central hydrogen burning; Case C: mass transfer after the end of central helium burning.
}

We describe our computational method in Sect. 2, and illustrate our binary model computation at the example of two representative cases in Sect. 3. In Sect. 4, we present the results of 74 model systems and discuss the formation of contact as a function of the main system parameters and the applied stellar physics. We discuss our results in Sect. 5, attempt a comparison with observations in Sect. 6, and summarise our main conclusions in Sect. 7.

\section{Computational methods}

We computed the evolution of massive close binary systems using a computer code generated by Braun (1997) on the basis of an implicit hydrodynamic stellar evolution code for single stars (cf. Langer 1991, 1998). It invokes the simultaneous evolution of the two stellar components of a binary and computes mass transfer within the Roche approximation (Kopal 1978).

Mass loss from the Roche lobe filling component through the first Lagrangian point is computed according to Ritter (1988) as

$\dot{M}=\dot{M}_{0} \exp \frac{R-R_{\mathrm{L}}}{H_{\mathrm{P}}} \quad$ with $\quad \dot{M}_{0}=\frac{1}{\sqrt{e}} \rho v_{\mathrm{s}} Q$.

$H_{\mathrm{P}}$ is the photospheric pressure scale height, $\rho$ the density, $v_{\mathrm{s}}$ the velocity of sound and $Q$ the effective crosssection of the stream through the first Lagrange pint according to Meyer \& Meyer-Hofmeister (1983). The distance between the first Lagrange point and the center of mass which enter $H_{\mathrm{P}}$ and $Q$ are interpolated from the tables of Kopal (1978) and Mochnacki (1984). We note that this treatment is only correct for stars with small photospheric pressure scale compared to their radius (Pastetter \& Ritter 1989). Otherwise, models of Paczyński \& Sienkiewicz (1972), Plavec et al. (1973), Savonije (1978) or Kolb \& Ritter (1990) might be used. However, this is not required for the binaries considered in this work.

Due to the exponential dependence of the mass transfer rate on the stellar radius, an explicit scheme for computing the mass transfer rate can easily lead to numerical instabilities. Thus, we derive the mass transfer rate implicitly using an iterative scheme (combined Secant/Bisection-method; cf. Press et al. 1988), which is numerically more stable (Braun 1997). However, for each iteration a complete stellar model needs to be computed.

The change of the orbital period due to the mass transfer and stellar wind mass loss is computed according to Podsiadlowski et al. (1992), with the specific angular momentum of the stellar wind material being determined according to Brookshaw \& Tavani (1993). The spin angular momentum of both stars is neglected. Mass transfer is treated quasi conservatively. The only mass loss from the system is due to the stellar winds of both components. The adopted stellar wind mass loss rates are described in Wellstein \& Langer (1999).

We include time-dependent thermohaline mixing - i.e. mixing in radiatively stable regions which occurs due to 
an outwards increase of the mean molecular weight. This mixing process is important in stars which accrete heliumenriched material. A time-dependent treatment of thermohaline mixing is important for identifying the borderline between contact and contact-free close binary systems as it occurs on the thermal time scale of the accreting star, which - at the borderline of contact-free systems is just equal to the mass transfer time scale. I.e., a time dependent treatment is required to reproduce the radius evolution of the accreting star during the mass transfer phase correctly as soon as helium-enriched material is being transferred (cf., Braun 1997).

Numerically, we treat thermohaline mixing through a diffusion scheme (Braun 1997). The corresponding diffusion coefficient is based on the work of Stern (1960), Ulrich (1972), and Kippenhahn et al. (1980); it reads

$D_{\mathrm{thm}}=-\alpha_{\mathrm{thm}} \frac{3 K}{2 c_{\mathrm{P}} \rho} \frac{\frac{\phi}{\delta} \nabla_{\mu}}{\nabla_{\mathrm{ad}}-\nabla}$,

where $K=4 a c /(3 \kappa \rho), \phi=(\partial \ln \rho / \partial \ln \mu)_{P, T}$, $\delta=-(\partial \ln \rho / \partial \ln T)_{P, \mu}, \quad \nabla_{\mu}=\mathrm{d} \ln \mu / \mathrm{d} \ln P, \quad \nabla_{\mathrm{ad}}=$ $(\partial \ln T / \partial \ln P)_{\mathrm{ad}}$, and $\nabla=\mathrm{d} \ln T / \mathrm{d} \ln P$. The quantity $\alpha_{\mathrm{thm}}$ is a efficiency parameter of order unity, and we use $\alpha_{\mathrm{thm}}=1$ throughout this paper.

The entropy of the accreted material is assumed to be equal to that of the surface of the mass gainer and the gravitational energy release due to mass transfer is treated as in Neo et al. (1977). Convection and semiconvection are treated as described in Langer (1991) and Braun \& Langer (1995) (cf. also Langer et al. 1983). A semiconvective efficiency parameter of $\alpha_{\mathrm{sc}}=0.01$ is applied in most models. We do not include so called convective core overshooting in our models, as recent evidence implies that the physical effect of increased convective core masses in massive main sequence stars may be due to effects of rapid rotation rather than convection (Maeder 1987; Langer 1992; Heger et al. 2000). As the rotation rates in close binaries may be reduced due to orbit circularisation and spin-orbit synchronisation (e.g., Savonije \& Papaloizou 1997), it is unclear to what extent any convective core mass increase would occur in those objects. Opacities are taken from Iglesias \& Rogers (1996). Changes in the chemical composition are computed using a nuclear network including the pp-chains, the CNO-tri-cycle, and the major helium-, carbon, neon and oxygen burning reactions. Further details about the computer program and input physics can be found in Langer (1998) and Wellstein \& Langer (1999). For all models, a metallicity of $2 \%$ is adopted.

\section{Case $A$ and $B$ evolution: Examples}

We have computed 74 Case A and Case B systems to derive the limits of contact free evolution for massive close binaries within the assumptions made in Sect. 2. Before we present the results of these models, we want to discuss the evolution of one typical Case A system (No. 31; cf. Sect. 4 below), and one Case B system (No. 37) in the following, to illustrate the capabilities of our method and the type and quality of the derived results. Those two systems are picked to show one detailed example per considered initial primary star mass - i.e., $12 M_{\odot}$ and $16 M_{\odot}$; we have discussed details of models with $25 M_{\odot}$ initial primary mass in Wellstein \& Langer (1999). Furthermore, the two examples shown in detail below correspond to systems which evolve contact-free. Therefore, we could follow the evolution of both binary components until the end of carbon burning (where we stopped the calculations).

\subsection{Case $A$}

Table 1 and Fig. 1 describe the evolution of our Case A system No. 31 (cf. Table 3 below), which initially consists of a $12 M_{\odot}$ primary $^{2}$ and a $7.5 M_{\odot}$ secondary star in a $2.5 \mathrm{~d}$ orbit. Mass transfer starts at a system age of $\sim 1.310^{7} \mathrm{yr}$, at a core helium mass fraction of the primary of 0.89 . The mass transfer rate rises to some $10^{-4} M_{\odot} \mathrm{yr}^{-1}$ during the so called rapid Case A mass transfer phase (Fig. 2), i.e. to values of the order of $M_{1} / \tau_{\mathrm{KH}, 1}, M_{1}$ being the primary mass and $\tau_{\mathrm{KH}, 1}$ the thermal time scale of the primary. The reason is that the orbital separation of both stars decreases until both components have the same mass, here about $9.75 M_{\odot}$. During the slow Case A mass transfer phase, the mass transfer is driven by the nuclear evolution of the primary, and the mass transfer rate drops to some $10^{-7} M_{\odot} \mathrm{yr}^{-1}$, i.e. values of the order of $M_{1} / \tau_{\text {nuc }, 1}$.

It is remarkable that the mass transfer does not immediately relax at the time of minimum orbital separation. In fact, Fig. 2 shows that only a small amount of mass $\left(\sim 0.15 M_{\odot}\right)$ is transfered during that slow Case A mass transfer, i.e. that its rapid phase does not stop until the primary mass is as small as $4 M_{\odot}$. The reason is that, in contrast to the rule of thumb that stars with radiative envelopes shrink upon mass loss, the evolved core hydrogen burning $12 M_{\odot}$ star has a negative mass-radius exponent, i.e. its thermal equilibrium radius increases for decreasing mass as a response to the increasing core-mass/envelopemass ratio. The thermally unstable mass transfer during the rapid Case $\mathrm{A}$ is ended only when the mass ratio becomes sufficiently small (cf. Ritter 1988).

During the rapid phase of the Case A mass transfer the primary becomes underluminous and the secondary becomes overluminous, due to the rapid mass loss and gain, respectively. I.e., they deviate by up to a factor of two from their thermal equilibrium luminosity (see the kinks in the evolutionary tracks towards the end of the Case A mass transfer in Fig. 1). During the slow phase of the Case A mass transfer, primary and secondary relax to thermal equilibrium. Due to the change in total mass, the secondary becomes more luminous than the primary, even

\footnotetext{
2 We designate the initially more massive star in the system as the primary, also during its later evolution when it has become the less massive and/or less luminous star. The other star is designated as the secondary.
} 


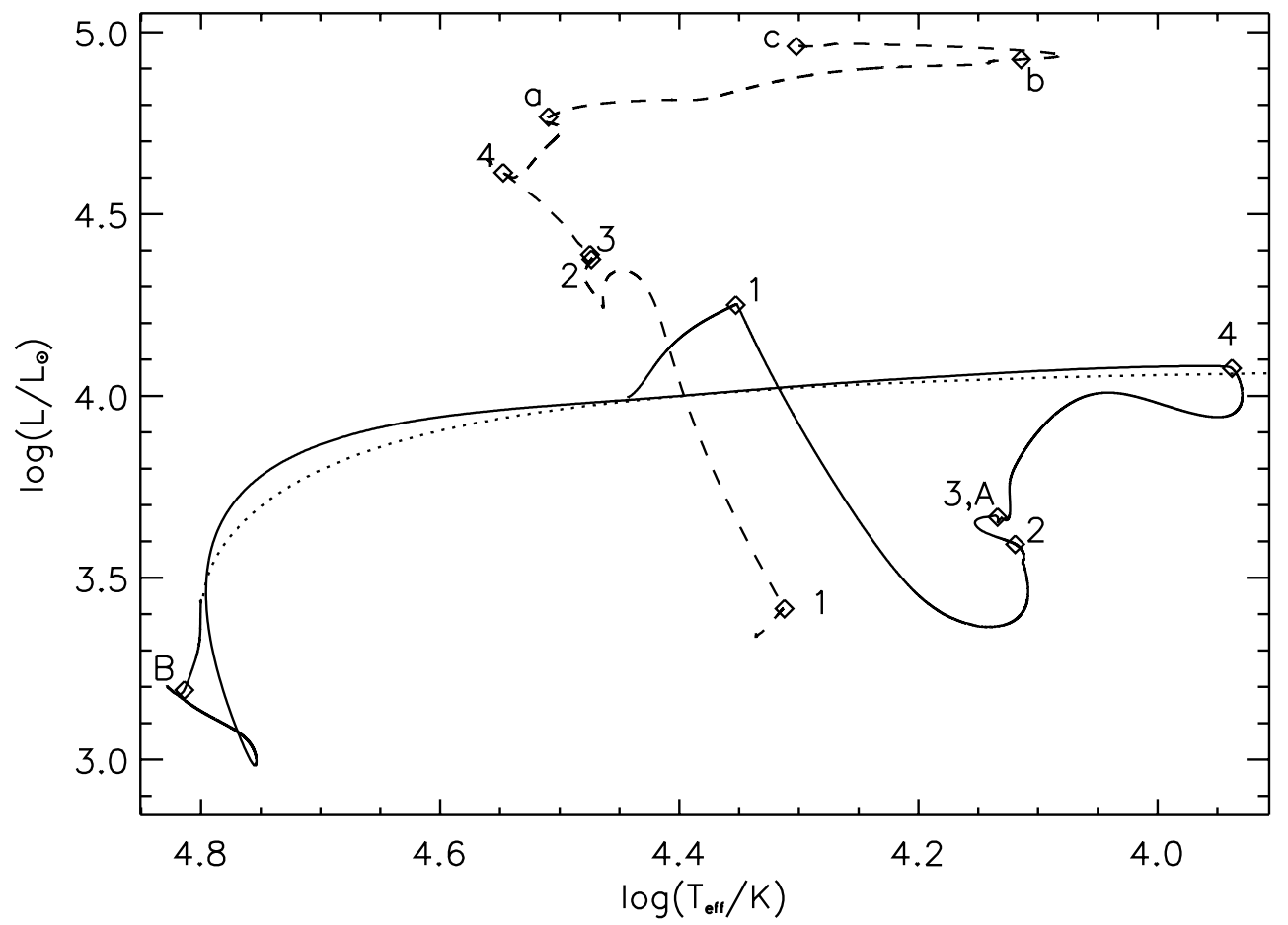

Fig. 1. Evolutionary tracks of the primary (solid and dotted line) and secondary star (dashed line) of our case A binary system No. 31 (initial masses are $12 M_{\odot}$ and $7.5 M_{\odot}$, the initial period is $2.5 \mathrm{~d}$; cf. Table 3 in Sect. 4) in the HR diagram. Beginning and the end of the mass transfer phases are marked with numbers; 1: begin of Case A, 2: end of Case A, 3: begin of Case AB, 4: end of Case AB. The labels A/a designate the end of central hydrogen burning of the primary/secondary, B/b the end of central helium burning of the primary/secondary, and c the point of the supernova explosion of the secondary. In this system, the secondary star ends its evolution first. The time of its supernova explosion marks the end of the solid line in the track of the primary. The further evolution of the primary is shown as dotted line. During this phase, it is treated as a single star since the system is likely broken up due to the secondary's explosion

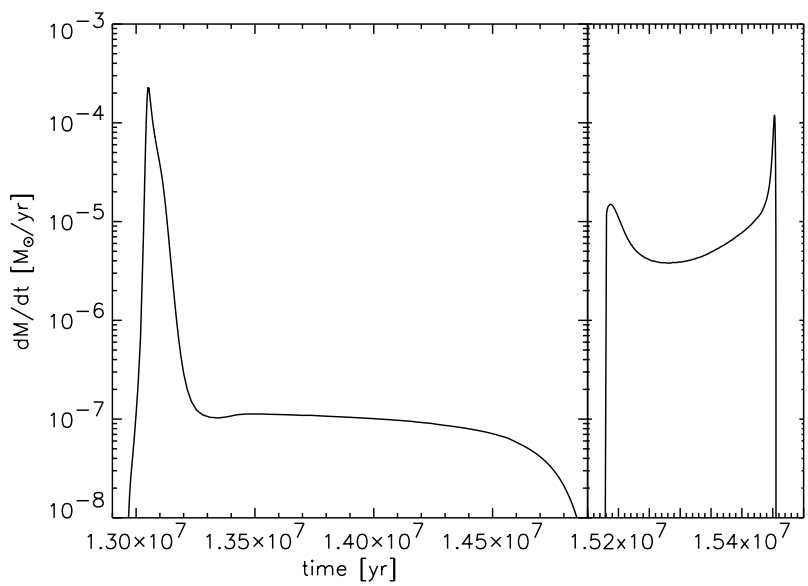

Fig. 2. Mass transfer rate as function of time for our Case A system No. 31 (initial masses are $12 M_{\odot}$ and $7.5 M_{\odot}$, the initial period is $2.5 \mathrm{~d}$; cf. Table 3 in Sect. 4) The left panel shows the rapid and slow Case A mass transfer, the right panel shows the ensuing Case $\mathrm{AB}$ mass transfer

though the primary, after the mass transfer, is strongly overluminous, i.e. its luminosity exceeds that of a $4 M_{\odot}$ single star by a factor of 10 .

At core hydrogen exhaustion, the primary mass is about $3.9 M_{\odot}$. As it still contains a hydrogen-rich enve-
Table 1. Evolutionary parameters of the Case A system No. 31. The calculation ends after core carbon exhaustion of the primary (note that the secondary star produces the first supernova in the system). The different columns have the following meaning: evolutionary stage $(\mathrm{ZAMS}=$ zero age main sequence; $\mathrm{ECHB}=$ end core hydrogen burning, $\mathrm{ECHeB}=$ end core helium burning; ECCB = end core carbon burning), evolutionary age (ZAMS: $t=0$ ), primary and secondary star mass, orbital period, primary and secondary star orbital velocity

\begin{tabular}{l|rrrrrr}
\hline & $\begin{array}{r}\text { time } \\
\text { Myr }\end{array}$ & $M_{\odot}$ & $M_{\odot}$ & $M_{\odot}$ & $\begin{array}{r}P \\
\mathrm{~d}\end{array} \mathrm{~km} \mathrm{~s}^{-1}$ & $\begin{array}{r}v_{2} \\
\mathrm{~km} \mathrm{~s}^{-1}\end{array}$ \\
\hline ZAMS & 0 & 12.0 & 7.50 & 2.5 & 162 & 260 \\
begin Case A & 12.96 & 11.9 & 7.49 & 2.52 & 162 & 258 \\
end Case A & 14.93 & 3.92 & 15.5 & 7.99 & 228 & 57.8 \\
ECHB primary & 15.17 & 3.92 & 15.5 & 7.99 & 228 & 57.8 \\
begin Case AB & 15.17 & 3.92 & 15.5 & 7.99 & 228 & 57.8 \\
end Case AB & 15.46 & 1.45 & 17.8 & 102.6 & 113 & 9.2 \\
ECHB secondary & 18.94 & 1.45 & 17.7 & 104.2 & 112 & 9.2 \\
ECHeB primary & 20.55 & 1.45 & 17.4 & 108.0 & 110 & 9.2 \\
ECHeB secondary & 20.82 & 1.45 & 17.2 & 108.1 & 109 & 9.2 \\
ECCB secondary & 21.16 & 1.45 & 17.0 & 108.4 & 109 & 9.2 \\
ECCB primary & 22.14 & 1.45 & - & - & - & - \\
\hline
\end{tabular}

lope, a hydrogen burning shell ignites and the star attempts to expand to red giant dimensions. This initiates the so called Case AB mass transfer, which proceeds on 
the thermal time scale of the primary and produces correspondingly high mass transfer rates (cf. Fig. 2). It leads to the loss of almost all remaining hydrogen and to the extinction of the hydrogen burning shell. The Case $A B$ mass transfer reduces the primary mass to about $1.45 M_{\odot}$, which corresponds to the mass of the convective hydrogen burning core at central hydrogen exhaustion. Due to the extreme mass ratio (cf. Table 1 ) the orbital period grows from $8 \mathrm{~d}$ to about $100 \mathrm{~d}$ during the Case $\mathrm{AB}$ mass transfer. The primary spends about 5 Myr for core helium burning, during which it remains on the helium-main sequence in the HR diagram (cf. Fig. 1). The ensuing helium shell burning expands the star to red giant dimensions, and a so called Case ABB mass transfer would occur (Kippenhahn \& Thomas 1979) - it does occur in other systems of our sample - if the secondary star would not have exploded in the meantime (see below). We follow the primary's evolution until core carbon exhaustion. Due to its small final mass, it is unlikely to produce a supernova explosion. Instead, mass loss during its red giant stage at moderate rates would suffice to substantially reduce the total mass before the core mass can grow to ignite neon burning (Habets 1986a,b; Woosley et al. 1995). I.e., our primary star of initially $12 M_{\odot}$ ends as an isolated $\mathrm{ONeMg}$-white dwarf.

The secondary star grows to $17.81 M_{\odot}$ and finishes core hydrogen burning about 3.5 Myr after the end of the case $\mathrm{AB}$ mass transfer. At this time it begins to expand to become a supergiant. However, due to the presence of a strong mean molecular weight barrier above the convective core at the onset of the mass transfer, it does not rejuvenate during core hydrogen burning, i.e. it does not adapt its convective core mass to its new total mass (cf. Hellings 1983, 1984; Braun \& Langer 1995). Therefore, the secondary star has, after the mass transfer, a much smaller helium core to envelope mass ratio as a single star of the same mass. For this reason, it remains in the blue part of the HR diagram and does never become a red supergiant (Braun \& Langer 1995).

Whether or not a secondary star rejuvenates depends strongly on the - still poorly known - efficiency of semiconvection mixing (Langer et al. 1983; Braun \& Langer 1995). Were rejuvenation occurring in our secondary, it would, after the mass transfer, evolve similar to a single star of $\sim 18 M_{\odot}$ and attempt to become a red supergiant after core hydrogen exhaustion. This would lead to a reverse Case B mass transfer in this system, with the consequence of contact evolution and a likely merging of both stars, due to the extreme mass ratio (cf. System No. 25 below, and Sect. 5.2, for more details).

Because the secondary star has a small helium core mass, its fuel supply for core helium burning is small. However, due to its comparatively large total mass, its luminosity is large. Both factors together imply a very short core helium burning life time of only 2 Myr. As a consequence, the secondary overtakes the primary in the evolutionary status and ends its evolution first. It explodes as a nitrogen-rich blue supergiant with properties similar to those of the progenitor of SN 1987A. It is helium- and nitrogen enriched due to the accretion, by factors 1.26 and 5.4 , respectively, while carbon and oxygen are depleted by factors of 0.23 and 0.81 (cf. Wellstein \& Langer 2001). Its core still has a memory of the lower initial mass: the helium core mass is only $\sim 4 M_{\odot}$ at core carbon exhaustion. This model applied to SN 1987A would predict the existence of a $1.45 M_{\odot}$ helium star with a luminosity of $\sim 10^{3} L_{\odot}$ and a temperature of $\sim 55000 \mathrm{~K}$ in the supernova remnant.

In many of our Case A systems - always for those with a $12 M_{\odot}$ primary - the secondary is the first star which reaches the supernova stage. This supernova reversal is discussed in more detail in Sect. 5.2.

\subsection{Case $B$}

Table 2 and Fig. 3 describe the evolution of our case B system No. 37 (cf. Table 3), starting with a $16 M_{\odot}$ primary and a $15 M_{\odot}$ secondary in an $8 \mathrm{~d}$ orbit. As the primary expands on a thermal time scale after core hydrogen exhaustion, the Case B mass transfer occurs on the thermal time scale of the primary, with mass transfer rates of the order of $M_{1} / \tau_{\mathrm{KH}, 1}$ (cf. Sect. 3.1). Both stars are far from thermal equilibrium during the whole mass transfer phase. However, also in this case, a fast and a somewhat slower Case B mass transfer phase can be distinguished (Fig. 4), since the orbit shrinks at first and expands after the mass ratio is reversed.

In the considered system, the mass transfer achieves about $210^{-3} M_{\odot} \mathrm{yr}^{-1}$ during the Case B mass overflow. This implies an accretion time scale of the secondary of the same order as its Kelvin-Helmholtz time scale. Consequently, the secondary swells considerably during the Case B mass transfer. While here, contact is not quite achieved, for an initial period of $9 \mathrm{~d}$ instead of $8 \mathrm{~d}$ the primary's radius at the onset of mass transfer is slightly larger, the mass transfer rate as well, and the system does evolve into contact at this stage (cf. system No. 39 in Sect. 4 below).

After the Case B mass transfer, very little hydrogen is left in the primary star. It evolves quickly into a hot and compact helium star of $\sim 3.8 M_{\odot}$, as which it burns helium in its core for about $1 \mathrm{Myr}$. Thereafter, it expands again (cf. Sect. 3.1) and fills its Roche lobe a second time, which initiates the so called case BB mass transfer. During this phase, it loses the major fraction of its helium envelope - $0.49 M_{\odot}$ - to the secondary. This mass transfer occurs again on the thermal time scale of the primary (cf. Fig. 4). Between core carbon exhaustion and neon ignition, the mass transfer ends. During the further evolution the binary system remains detached, but the primary remains an extended supergiant and nearly fills its Roche lobe. In the final state, the once $16 M_{\odot}$ primary is a $2.3 M_{\odot}$ helium giant with a remaining helium mass of $0.74 M_{\odot}$. The core of the primary will collapse and produce a supernova explosion. The explosion characteristics may 


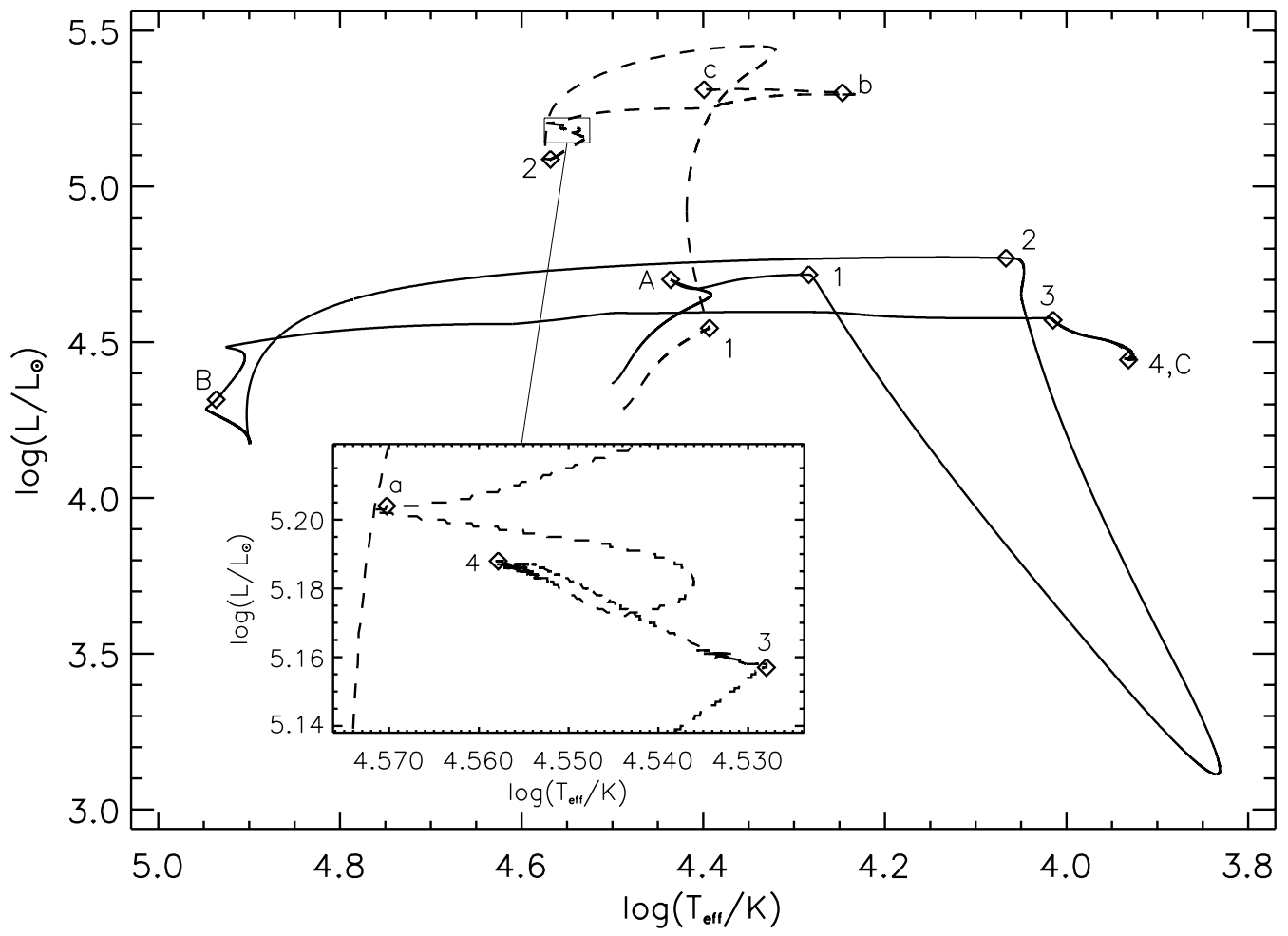

Fig. 3. Evolutionary tracks of the primary (solid and dotted line) and secondary star (dashed line) of our case B binary system No. 37 (initial masses are $16 M_{\odot}$ and $15 M_{\odot}$, the initial period is $8 \mathrm{~d}$; cf. Table 3 in Sect. 4 ) in the HR diagram. Beginning and end of the mass transfer phases are marked with numbers; 1 : beginning of case B, 2: end of case B, 3: beginning of case BB, 4: end of case BB. The labels A/a designate the end of central hydrogen burning of the primary/secondary, B/b the end of central helium burning of the primary/secondary, and $\mathrm{C} / \mathrm{c}$ the point of their supernova explosion

Table 2. Evolutionary parameters of the Case B system No. 37. Both stars are followed beyond core carbon exhaustion. The different columns have the following meaning: evolutionary stage $(\mathrm{ZAMS}=$ zero age main sequence; $\mathrm{ECHB}=$ end core hydrogen burning, $\mathrm{ECHeB}=$ end core helium burning; $\mathrm{ECCB}$ $=$ end core carbon burning), evolutionary age (ZAMS: $t=0)$, primary and secondary star mass, orbital period, primary and secondary star orbital velocity

\begin{tabular}{l|rrrrrr}
\hline & time & $M_{1}$ & $M_{2}$ & $P$ & $v_{1}$ & $\begin{array}{r}v_{2} \\
\text { Myr }\end{array}$ \\
& $M_{\odot}$ & $M_{\odot}$ & $\mathrm{d}$ & $\mathrm{km} \mathrm{s}^{-1}$ & $\mathrm{~km} \mathrm{~s}^{-1}$ \\
\hline ZAMS & 0 & 16.0 & 15.0 & 8 & 162 & 173 \\
ECHB primary & 9.472 & 15.8 & 14.8 & 8.19 & 160 & 170 \\
begin Case B & 9.490 & 15.8 & 14.8 & 8.19 & 160 & 170 \\
end Case B & 9.513 & 3.83 & 26.7 & 94.9 & 128 & 18.3 \\
ECHeB primary & 10.46 & 3.61 & 26.5 & 99.1 & 126 & 17.1 \\
begin Case BB & 10.68 & 2.81 & 26.5 & 102.9 & 126 & 13.4 \\
ECCB primary & 10.68 & 2.33 & 26.8 & 170.7 & 109 & 9.4 \\
end Case BB & 10.68 & 2.32 & 26.8 & 172.1 & 108 & 9.4 \\
ECHB secondary & 10.95 & - & 26.7 & - & - & - \\
ECHeB secondary & 11.71 & - & 26.0 & - & - & - \\
ECCB secondary & 11.83 & - & 25.9 & - & - & - \\
\hline
\end{tabular}

resemble those of types $\mathrm{Ib}$ or Ic supernovae (Shigeyama et al. 1990; Woosley et al. 1995). However, the helium envelope of our model star is very extended; its radius is $\sim 80 R_{\odot}$. Theoretical models for the explosions for such cool helium giants are still missing in the literature. Due to the large final mass ratio of $27 / 2.3$, this system has a

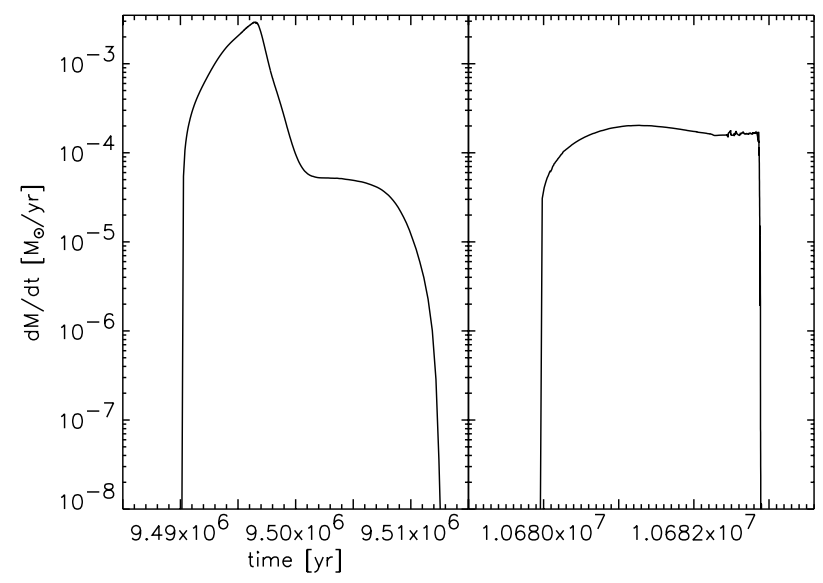

Fig. 4. Mass transfer rate as function of time for our Case B system No. 37 (initial masses are $16 M_{\odot}$ and $15 M_{\odot}$, the initial period is $8 \mathrm{~d}$; cf. Table 3 in Sect. 4) The left panel shows the Case $B$ mass transfer, the right panel shows the ensuing Case BB mass transfer

good chance to remain bound after the explosion of the primary component.

The secondary star grows to almost $27 M_{\odot}$ during the Case B mass transfer. It continues its main sequence evolution thereafter and is still burning hydrogen in its core when the primary explodes. During the Case BB mass transfer, it accretes $0.49 M_{\odot}$ of almost pure helium from 


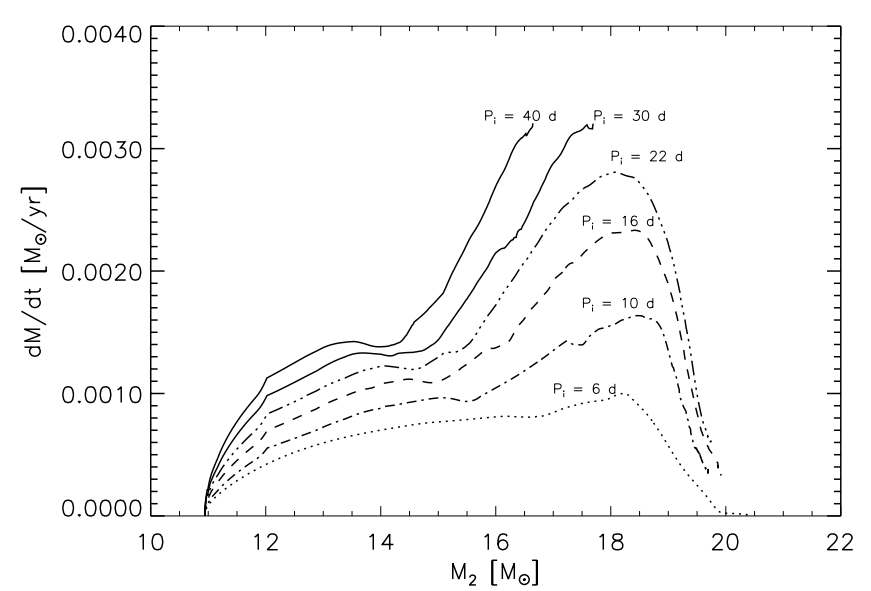

Fig. 5. Mass transfer rates as a function of secondary mass for Case B systems initially consisting of a $12 M_{\odot}$ and a $11 M_{\odot}$ star with initial periods in the range $6 \ldots 40 \mathrm{~d}$ (Nos. 3, 4, 5, 6, 7 and 8). The systems with 30 and 40 days initial period form contact, at which time we stop the calculation

the primary. During this phase, it becomes very helium and nitrogen rich at the surface. Even though during and after the Case BB mass transfer this enrichment is strongly diluted by thermohaline mixing, the final helium and nitrogen enrichment factors are 1.25 and 4 , i.e. the final surface mass fractions are 0.35 and 0.0041 for helium and nitrogen, respectively.

After core hydrogen exhaustion, the secondary expands, but it remains a blue supergiant up to its supernova explosion (Fig. 3) due to the lack of rejuvenation (cf. Sect. 3.1). The explosion of the secondary disrupts the binary system with a high probability.

\section{Formation of contact}

In this section, we present results for 74 computed systems. Rather than providing a detailed description of the evolution of each system, we focus on the general trends of key properties as function of the major initial parameters. Table 3 provides the most important quantities for all computed systems. We focus on the various mechanisms which can drive massive close binary systems into contact.

\subsection{Contact formation due to small or large periods}

\subsubsection{Case B}

Case B systems with an initial mass ratio close to 1 and a rather short initial period (early Case B) are known to have a good chance to avoid contact during the Case B mass transfer (cf. Pols 1994). Our Table 3 contains 15 examples, i.e. systems Nos. 2...6, 12, 16, 18...20, 27, 28 (initial primary mass of $12 M_{\odot}$ ), and Nos. 35, 37 and 44 (initial primary mass of $16 M_{\odot}$ ). The fact that no such system with an initial primary mass of $25 M_{\odot}$ could be found is discussed in Sect. 5.1. It is often assumed that late Case B systems, i.e. such with a relatively large initial period, evolve through a common envelope stage

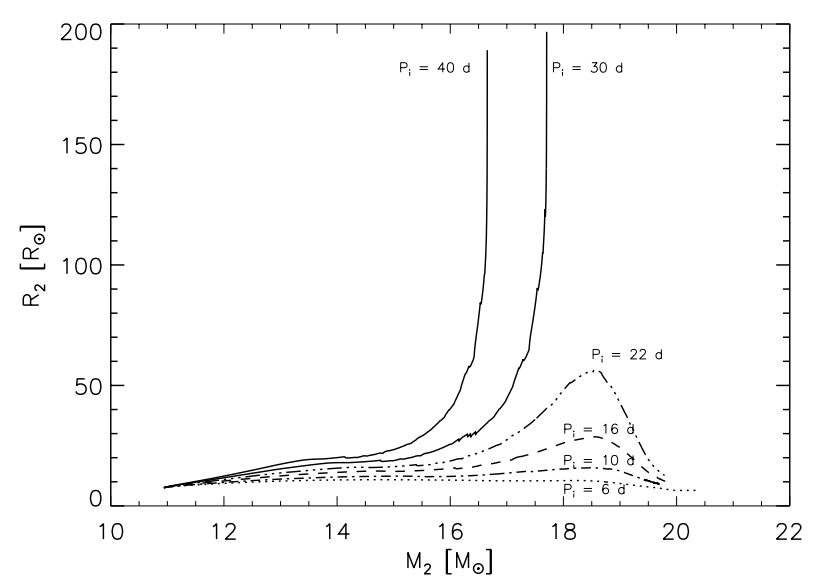

Fig. 6. Radius of the secondary during mass transfer as a function of its mass, for the same systems as in Fig. 5. In the systems with 30 and 40 days initial period the large increase in radius leads to contact, at which time we stop the calculation

(Podsiadlowski et al. 1992; Vanbeveren 1998a,b,c). In the following, we will have a closer look to the transition region in between both extremes.

Figure 5 shows the mass transfer rates as a function of the secondary mass for Case B binaries with initial primary and secondary masses of $12 M_{\odot}$ and $11 M_{\odot}$, respectively, but different initial periods in the range $6 \ldots 40 \mathrm{~d}$ (Systems Nos. 3 to 8). Generally, initially wider systems develop larger mass transfer rates. However, it is interesting to consider the time dependence of the mass transfer rate of these systems in some detail. The first maximum in the mass transfer rate is related to the end of decrease of the orbital separation at mass ratio one. I.e., when the primary has become the less massive component in the system, the period and the Roche lobe of the primary begin to grow, and the increase of the mass transfer rate flattens. This happens with some time delay due to the finite thermal response time of the primary.

Later during the mass transfer, the orbit widens significantly. Therefore, the primary becomes more extended and its surface temperature decreases. In the wider systems considered in Fig. 5, the outer envelope of the primary becomes even convectively unstable. It is the drop of the adiabatic mass-radius exponent $\zeta_{\text {ad }}$ (cf. Ritter 1988) which leads to another rise of the mass transfer rate even though the orbit widens. We designate this feature as delayed contact. Table 3 shows nine systems which evolve delayed contact. It is restricted to the Case B and has no counterpart in our Case A systems (cf. Sect. 4.1.2).

Figure 6 displays the radius evolution of the secondary stars of the systems considered in Fig. 5. Initially, the radii do not grow significantly, although it can be seen seen that larger radii are obtained for larger initial periods, i.e. for larger mass transfer rates. Later on, mass transfer rates in excess of $\sim 310^{-3} M_{\odot} \mathrm{yr}^{-1}$ make the secondary swell enormously. This mass transfer rate corresponds roughly to $\dot{M}=R L /(G M)$, i.e. to the condition $M / \dot{M}=G M^{2} /(R L)$ for the secondary. In the two systems with initial periods 
Table 3. Characteristic quantities for all computed systems: system number, initial values of primary mass $M_{1}$, secondary mass $M_{2}$, and period $P_{i}$, and the mass transfer case. Bold values for the initial masses identify the component which ends its evolution first. Further columns are: the kind of contact experienced by the system (no contact = "-", premature contact $=$ "M", reverse contact $=$ "R", $q$-contact $=$ "Q", and delayed contact $=$ "D"; a lower case letter is used if the secondary fills less than 1.5 times its Roche lobe; systems Nos. 52 and 59 experience a second contact after a weak $q$-contact), the core helium mass fraction of the primary at the onset of the first mass transfer $Y_{1}$, and $M_{1}^{\prime}, M_{2}^{\prime}, L_{1}^{\prime}, L_{2}^{\prime}, R_{1}^{\prime}, R_{2}^{\prime}, P^{\prime}$ denote primary and secondary mass, luminosity, effective temperature, radius and orbital period after the Case $\mathrm{A} / \mathrm{AB}$ or Case $\mathrm{B}$ mass transfer, at a time when the core helium mass fraction of the primary has decreased to 0.8 due to helium burning. $\dot{M}_{\max }$ is the maximum mass transfer rate (lower limits are give for contact systems, values in italic are uncertain) and $\Delta M$ is the amount of mass transfered by the time the maximum mass transfer rate or contact is reached. $\beta$ designates the fraction of the transfered matter that is accreted, assuming no further accretion after the secondary overfills its Roche lobe more than 1.5 times

\begin{tabular}{|c|c|c|c|c|c|c|c|c|c|c|c|c|c|c|c|c|}
\hline No. & $M_{1}$ & $M_{2}$ & $\begin{array}{l}P_{i} \\
\mathrm{~d}\end{array}$ & case & & $\begin{array}{l}Y_{1} \\
\%\end{array}$ & $M_{1}^{\prime}$ & $M_{2}^{\prime}$ & $\begin{array}{c}L_{1}^{\prime} \\
\quad \log (\end{array}$ & $\begin{array}{r}L_{2}^{\prime} \\
\odot)\end{array}$ & $R_{1}^{\prime}$ & $\begin{array}{r}R_{2}^{\prime} \\
R_{\odot}\end{array}$ & $\begin{array}{l}P^{\prime} \\
\mathrm{d}\end{array}$ & $\begin{array}{c}\dot{M}_{\max } \\
\frac{10^{-4} M_{\odot}}{\mathrm{yr}}\end{array}$ & $\begin{array}{c}\Delta M \\
M_{\odot}\end{array}$ & $\beta$ \\
\hline 1 & 12 & 11.5 & 2.5 & A & - & 87 & 1.42 & 21.5 & 2.98 & 4.99 & 0.3 & 16.5 & 218 & 0.71 & 1.8 & 1.00 \\
\hline 2 & 12 & 11 & 3 & B & - & 98 & 2.44 & 20.4 & 3.93 & 4.74 & 0.8 & 7.2 & 54 & 4.0 & 7.3 & 1.00 \\
\hline 3 & 12 & 11 & 6 & B & - & 98 & 2.38 & 20.4 & 3.82 & 4.79 & 0.6 & 6.8 & 115 & 10 & 7.3 & 1.00 \\
\hline 4 & 12 & 11 & 10 & B & - & 98 & - & - & - & - & - & - & - & 17 & 7.5 & 1.00 \\
\hline 5 & 12 & 11 & 16 & B & - & 98 & - & - & - & - & - & - & - & 25 & 7.5 & 1.00 \\
\hline 6 & 12 & 11 & 22 & B & - & 98 & - & - & - & - & - & - & - & 28 & 7.6 & 1.00 \\
\hline 7 & 12 & 11 & 30 & B & $\mathrm{D}$ & 98 & - & - & - & - & - & - & - & $>33$ & 6.5 & 0.68 \\
\hline 8 & 12 & 11 & 40 & B & $\mathrm{D}$ & 98 & - & - & - & - & - & - & - & $>33$ & 5.7 & 0.60 \\
\hline 9 & 12 & 10.5 & 2 & A & $\mathrm{M}$ & 75 & - & - & - & - & - & - & - & 0.67 & 1.5 & 0.70 \\
\hline 10 & 12 & 10.5 & 2.5 & A & - & 87 & 1.42 & 20.7 & 2.97 & 4.87 & 0.3 & 7.9 & 190 & 0.93 & 2.0 & 1.00 \\
\hline 11 & 12 & 10 & 2 & A & $\mathrm{M}$ & 76 & - & - & - & - & - & - & - & 0.82 & 1.6 & 0.68 \\
\hline 12 & 12 & 10 & 16 & B & - & 98 & 2.43 & 19.4 & 3.81 & 4.71 & 0.6 & 6.6 & 255 & 22 & 7.0 & 1.00 \\
\hline 13 & 12 & 10 & 22 & B & $\mathrm{D}$ & 98 & - & - & - & - & - & - & - & $>28$ & 6.9 & 0.73 \\
\hline 14 & 12 & 9.5 & 2 & A & - & 76 & 1.29 & 19.4 & 2.93 & 5.00 & 0.4 & $32.3^{e}$ & 171 & 1.8 & 2.0 & 1.00 \\
\hline 15 & 12 & 9.5 & 2.5 & A & - & 88 & 1.41 & 19.7 & 2.96 & 4.79 & 0.3 & 7.5 & 166 & 1.2 & 2.0 & 1.00 \\
\hline 16 & 12 & 9.5 & 3.5 & B & - & 98 & 2.45 & 18.9 & 3.94 & 4.63 & 0.8 & 6.3 & 50 & 4.0 & 2.9 & 1.00 \\
\hline $17^{a}$ & 12 & 9 & 2 & A & $\mathrm{R}$ & 76 & 1.15 & 19.4 & 2.67 & 4.87 & 0.3 & 9.0 & 214 & 1.2 & 1.7 & 1.00 \\
\hline 18 & 12 & 9 & 3.5 & B & - & 98 & 2.32 & 18.5 & 3.74 & 4.63 & 0.6 & 6.5 & 53 & 6.5 & 3.4 & 1.00 \\
\hline 19 & 12 & 9 & 6 & B & - & 98 & 2.36 & 18.5 & 3.81 & 4.63 & 0.6 & 6.2 & 87 & 9.9 & 7.4 & 1.00 \\
\hline 20 & 12 & 9 & 10 & B & - & 98 & - & - & - & - & - & - & - & 15 & 7.0 & 1.00 \\
\hline 21 & 12 & 9 & 16 & B & $\mathrm{d}$ & 98 & - & - & - & - & - & - & - & 28 & 7.0 & 1.00 \\
\hline 22 & 12 & 9 & 22 & B & $\mathrm{D}$ & 98 & - & - & - & - & - & - & - & $>29$ & 5.9 & 0.62 \\
\hline 23 & 12 & 8.5 & 2.5 & A & - & 88 & 1.43 & 18.8 & 2.97 & 4.72 & 0.3 & 6.8 & 135 & 1.6 & 2.1 & 1.00 \\
\hline 24 & 12 & 8 & 2 & $\mathrm{~A}$ & - & 77 & 1.12 & 18.5 & 2.64 & 4.84 & 0.3 & 8.5 & 189 & 3.0 & 2.3 & 1.00 \\
\hline $25^{c}$ & 12 & 8 & 2.5 & $\mathrm{~A}$ & $\mathrm{R}$ & 87 & 1.66 & 17.9 & 3.18 & 4.68 & 0.4 & 6.5 & 81.5 & 3.6 & 2.5 & 1.00 \\
\hline 26 & 12 & 8 & 3.5 & B & $q$ & 98 & - & - & - & - & - & - & - & 7.3 & 3.0 & 1.00 \\
\hline 27 & 12 & 8 & 6 & B & - & 98 & 2.37 & 17.5 & 3.79 & 4.55 & 0.6 & 5.8 & 72 & 8.9 & 3.5 & 1.00 \\
\hline 28 & 12 & 8 & 10 & B & - & 98 & - & - & - & - & - & - & - & 15 & 6.8 & 1.00 \\
\hline 29 & 12 & 8 & 16 & B & $\mathrm{D}$ & 98 & - & - & - & - & - & - & - & $>24$ & 6.0 & 0.63 \\
\hline 30 & 12 & 7.5 & 2 & $\mathrm{~A}$ & $q$ & 78 & - & - & - & - & - & - & - & 2.1 & 2.0 & 1.00 \\
\hline 31 & 12 & 7.5 & 2.5 & A & - & 89 & 1.45 & 17.8 & 2.99 & 4.64 & 0.3 & 6.3 & 104 & 2.5 & 2.0 & 1.00 \\
\hline 32 & 12 & 7.5 & 3.5 & B & $\mathrm{Q}$ & 98 & - & - & - & - & - & - & - & $>8.1$ & 2.8 & 0.27 \\
\hline 33 & 12 & 6.5 & 2 & A & $\mathrm{Q}$ & 78 & - & - & - & - & - & - & - & $>4.6$ & 1.4 & 0.13 \\
\hline 34 & 16 & 15.7 & 3.2 & A & - & 91 & 2.79 & 27.8 & 3.88 & 5.14 & 0.5 & 9.3 & 75 & 3.5 & 3.6 & 1.00 \\
\hline 35 & 16 & 15.7 & 6 & B & - & 98 & 3.49 & 27.5 & 4.17 & 5.14 & 0.6 & 8.3 & 86 & 22 & 7.7 & 1.00 \\
\hline 36 & 16 & 15 & 2.5 & A & $\mathrm{M}$ & 80 & - & - & - & - & - & - & - & 2.5 & 2.6 & 0.70 \\
\hline 37 & 16 & 15 & 8 & B & - & 98 & 3.63 & 26.7 & 4.20 & 5.10 & 0.7 & 8.9 & 96 & 29 & 8.0 & 1.00 \\
\hline
\end{tabular}


Table 3. continued

\begin{tabular}{|c|c|c|c|c|c|c|c|c|c|c|c|c|c|c|c|c|}
\hline No. & $M_{1}$ & & $\begin{array}{l}P_{\mathrm{i}} \\
\mathrm{d}\end{array}$ & case & & $\begin{array}{l}Y_{1} \\
\%\end{array}$ & $\begin{array}{l}M_{1}^{\prime} \\
\quad\end{array}$ & $M_{2}^{\prime}$ & $\begin{array}{c}L_{1}^{\prime} \\
\log \end{array}$ & $\begin{array}{r}L_{2}^{\prime} \\
\left.L_{\odot}\right)\end{array}$ & $R_{1}^{\prime}$ & $R_{2}^{\prime}$ & $\begin{array}{l}P^{\prime} \\
\mathrm{d}\end{array}$ & $\begin{array}{c}\dot{M}_{\mathrm{m}} \\
\frac{10^{-4} M_{\odot}}{\mathrm{yr}}\end{array}$ & $\begin{array}{c}\Delta M \\
M_{\odot}\end{array}$ & $\beta$ \\
\hline 38 & 16 & 15 & 9 & B & $\mathrm{d}$ & 98 & 3.64 & 26.7 & 4.22 & 5.10 & 0.7 & 8.1 & 107 & 32 & 8.1 & 1.00 \\
\hline 39 & 16 & 15 & 15 & B & $\mathrm{D}$ & 98 & - & - & - & - & - & - & - & $>37$ & 6.7 & 0.48 \\
\hline 40 & 16 & 14 & 2 & A & $\mathrm{M}$ & 69 & - & - & - & - & - & - & - & 2.2 & 2.2 & 0.86 \\
\hline $41^{a}$ & 16 & 14 & 2 & A & - & 68 & 2.12 & 27.0 & 3.68 & 5.21 & 0.6 & 11.0 & 110 & 1.2 & 2.1 & 1.00 \\
\hline 42 & 16 & 14 & 2.5 & A & - & 80 & 2.39 & 26.5 & 3.78 & 5.17 & 0.6 & 11.0 & 97.5 & 3.1 & 2.9 & 1.00 \\
\hline 43 & 16 & 14 & 3 & A & - & 87 & 2.52 & 26.6 & 3.75 & 5.10 & 0.5 & 9.0 & 101 & 2.0 & 2.8 & 1.00 \\
\hline 44 & 16 & 14 & 6 & B & - & 98 & 3.60 & 25.8 & 4.19 & 5.04 & 0.6 & 7.8 & 67 & 24 & 7.9 & 1.00 \\
\hline 45 & 16 & 14 & 9 & B & $\mathrm{D}$ & 98 & 3.64 & 25.7 & 4.21 & 5.04 & 0.7 & 7.6 & 98 & 31 & 8.2 & $0.64^{f}$ \\
\hline 46 & 16 & 13 & 2 & A & $\mathrm{M}$ & 69 & - & - & - & - & - & - & - & 3.0 & 2.5 & 0.68 \\
\hline 47 & 16 & 13 & 2.5 & A & - & 80 & 2.28 & 26.1 & 3.65 & 5.11 & 0.5 & 9.1 & 99 & 2.1 & 2.4 & 1.00 \\
\hline $48^{c}$ & 16 & 13 & 2.5 & A & - & 79 & 2.47 & 25.6 & 3.77 & 5.10 & 0.6 & 7.6 & 78 & 4.3 & 3.0 & 1.00 \\
\hline 49 & 16 & 13 & 3 & A & - & 88 & 2.64 & 25.3 & 3.84 & 5.07 & 0.5 & 9.1 & 64 & 5.2 & 3.5 & 1.00 \\
\hline $50^{c}$ & 16 & 13 & 3 & A & - & 87 & 2.66 & 25.2 & 3.81 & 5.08 & 0.5 & 7.8 & 62,5 & 5.1 & 3.2 & 1.00 \\
\hline 51 & 16 & 13 & 4 & B & $\mathrm{d}$ & 98 & 3.55 & 24.8 & 4.19 & 4.99 & 0.7 & 8.2 & 41 & 18 & 6.9 & 1.00 \\
\hline 52 & 16 & 13 & 6 & B & D & 98 & 3.70 & 24.7 & 4.24 & 4.95 & 0.7 & 6.8 & 57 & 27 & 7.8 & $0.62^{f}$ \\
\hline 53 & 16 & 12 & 1.5 & A & $\mathrm{M}$ & 54 & - & - & - & - & - & - & - & 3.5 & 2.4 & 0.71 \\
\hline 54 & 16 & 12 & 1.7 & A & $\mathrm{M}$ & 61 & - & - & - & - & - & - & - & 3.7 & 2.5 & 0.75 \\
\hline 55 & 16 & 12 & 2 & A & - & 70 & 2.07 & 24.8 & 3.57 & 5.17 & 0.5 & 11.8 & 93 & 4.2 & 2.8 & 1.00 \\
\hline 56 & 16 & 12 & 4 & B & d & 98 & 3.55 & 23.9 & 4.17 & 4.93 & 0.6 & 7.0 & 37 & 18 & 6.3 & 1.00 \\
\hline 57 & 16 & 12 & 6.5 & B & D & 98 & - & - & - & - & - & - & - & 26 & 7.7 & $0.38^{f}$ \\
\hline 58 & 16 & 11 & 2 & A & - & 71 & 2.00 & 24.1 & 3.56 & 5.10 & 0.5 & 10.8 & 89 & 5.7 & 3.1 & 1.00 \\
\hline $59^{c}$ & 16 & 11 & 2 & A & $\mathrm{qR}$ & 69 & 2.10 & 24.0 & 3.54 & 5.13 & 0.5 & 8.4 & 77 & 5.9 & 3.1 & 1.00 \\
\hline 60 & 16 & 11 & 3 & A & - & 89 & 2.52 & 23.8 & 3.74 & 4.95 & 0.5 & 7.3 & 61 & 3.8 & 2.8 & 1.00 \\
\hline 61 & 16 & 11 & 3.2 & A & - & 92 & 2.81 & 23.3 & 3.87 & 4.93 & 0.5 & 7.4 & 42 & 8.2 & 4.0 & 1.00 \\
\hline 62 & 16 & 10 & 2.5 & A & $q$ & 81 & 2.28 & 23.1 & 3.77 & 4.97 & 0.6 & 7.8 & 65 & 8.7 & 3.5 & 1.00 \\
\hline 63 & 16 & 10 & 3 & B & $q$ & 89 & - & - & - & - & - & - & - & 9.9 & 3.9 & 1.00 \\
\hline 64 & 16 & 9 & 2.5 & A & $\mathrm{Q}$ & 82 & - & - & - & - & - & - & - & 12 & 3.4 & $0.16^{f}$ \\
\hline 65 & 25 & 24 & 3.5 & A & - & 83 & 5.15 & 40.6 & 4.53 & 5.54 & 0.6 & 13.7 & 41 & 3.0 & 4.6 & 1.00 \\
\hline $66^{a}$ & 25 & 24 & 3.5 & A & - & 83 & 5.21 & 40.6 & 4.54 & 5.54 & 0.6 & 12.4 & 40 & 3.0 & 4.2 & 1.00 \\
\hline $67^{b}$ & 25 & 24 & 3.5 & A & - & 82 & $\complement^{d}$ & 40.5 & $\complement^{d}$ & 5.54 & $\simeq^{d}$ & 11.2 & - & 3.0 & 4.6 & 1.00 \\
\hline 68 & 25 & 24 & 5 & B & $\mathrm{d}$ & 98 & 7.28 & 39.2 & 4.85 & 5.45 & 0.8 & 11.9 & 31 & 30 & 8.2 & 1.00 \\
\hline 69 & 25 & 24 & 9 & B & D & 98 & - & - & - & - & - & - & - & $>37$ & 7.4 & 0.43 \\
\hline 70 & 25 & 23 & 4 & A & - & 87 & 5.31 & 39.4 & 4.56 & 5.49 & 0.6 & 12.4 & 39 & 4.0 & 5.2 & 1.00 \\
\hline 71 & 25 & 22 & 2.5 & $\mathrm{~A}$ & - & 70 & 4.80 & 39.6 & 4.45 & 5.55 & 0.7 & 14.2 & 39 & 2.7 & 2.9 & 1.00 \\
\hline $72^{c}$ & 25 & 22 & 2.5 & A & - & 68 & 4.68 & 38.9 & 4.44 & 5.62 & 0.7 & 10.5 & 39 & 5.3 & 3.6 & 1.00 \\
\hline 73 & 25 & 19 & 4 & $\mathrm{~A}$ & - & 88 & 5.26 & 35.9 & 4.55 & 5.38 & 0.7 & 8.7 & 30 & 6.3 & 4.9 & 1.00 \\
\hline 74 & 25 & 16 & 4 & A & - & 89 & 5.22 & 33.3 & 4.54 & 5.29 & 0.6 & 8.8 & 24 & 8.8 & 5.0 & 1.00 \\
\hline
\end{tabular}

${ }^{a} \alpha_{\mathrm{sc}}=0.02$.

${ }^{b} \alpha_{\mathrm{sc}}=0.04$.

${ }^{c}$ Schwarzschild criterion instead of Ledoux criterion used.

${ }^{d}$ Only secondary is computed but primary should not be very different from Nos. 65 and 66 .

${ }^{e}$ Secondary at ECHeB.

$f$ Treated as if $\beta=1$.

of 30 and 40 days, the radius increases until the secondary This effect marks the limit of contact free systems towards also fills its Roche lobe and a contact system is formed. larger periods. 
It is remarkable that approximately $5 M_{\odot}$ for the $40 \mathrm{~d}$ binary (system No. 8 ) and $6 M_{\odot}$ for the $30 \mathrm{~d}$ binary (system No. 7) are transfered before the contact occurs (cf. Fig. 6). From Fig. 6 we see that contact occurs the earlier (in terms of already transfered mass) the larger the initial system period is. I.e., there is a continuous transition towards very wide binaries with convective primaries, which evolve into contact at the very beginning of the mass transfer process (Podsiadlowski et al. 1992).

According to the estimate of Webbink (1984), which compares the orbital energy of the binary with the binding energy of the envelope, our System No. 7 does not merge during the Case B mass transfer. Instead, with an efficiency parameter of $\alpha_{\mathrm{ce}}=1$, it obtains a period of about $7.5 \mathrm{~d}$ in a common envelope evolution by expelling the remaining envelope of the primary of $\sim 3 M_{\odot}$ from the system.

In summary, in delayed contact systems a conservative part of Case B mass transfer is followed by a nonconservative one, leading to an oscillation of the orbit: it first shrinks until the mass ratio is 1 , then widens up to the end of the conservative part, and then shrinks again due to mass and angular momentum loss from the system. For our System No. 7 , the parameter $\beta$ for the complete Case B mass transfer, i.e., the ratio of the amount of mass accreted by the secondary to the amount lost by the primary, is about $\beta \simeq 6 M_{\odot} / 9 M_{\odot} \simeq 0.67$.

After the delayed contact and common envelope evolution, System No. 7 consists of a $\sim 20 M_{\odot}$ main sequence star - the secondary - and a $\sim 2.4 M_{\odot}$ helium star in a $7.5 \mathrm{~d}$ orbit. The further evolution (which we have not computed) depends on the post-common envelope period. For $7.5 \mathrm{~d}$, the primary fills its Roche lobe as it expands to a helium giant, and transfers part of its helium envelope to the secondary in a Case BB mass transfer, similar to that discussed in Sect. 3.2 for system No. 37.

In contrast, the systems which avoid contact during the Case B mass transfer all together can often, even if the secondary evolves faster, avoid the reverse Case B mass transfer if the secondary star does not rejuvenate (cf. values of $T_{\text {eff,s }}^{\prime}$ in Table 3). After the Case B mass transfer, their periods are typically larger than $50 \mathrm{~d}$.

We find that in our most massive systems, with a primary mass of $25 M_{\odot}$, even early Case B mass transfer leads into contact, even for an initial mass ratios very close to 1 (cf. systems Nos. 68 and 69 in Table 3). In the two considered systems, large amounts of mass are transferred conservatively before contact occurs $\left(8.2 M_{\odot}\right.$ and $7.4 M_{\odot}$; cf. Table 3), i.e., we have a delayed contact situation. In system No. 68 , the contact is marginal, in system No. 69 it is not.

\subsubsection{Case $A$}

Figure 7 demonstrates a completely different mechanism of contact formation operating in Case A systems, which occurs for short periods (cf. also Pols 1994). It shows the

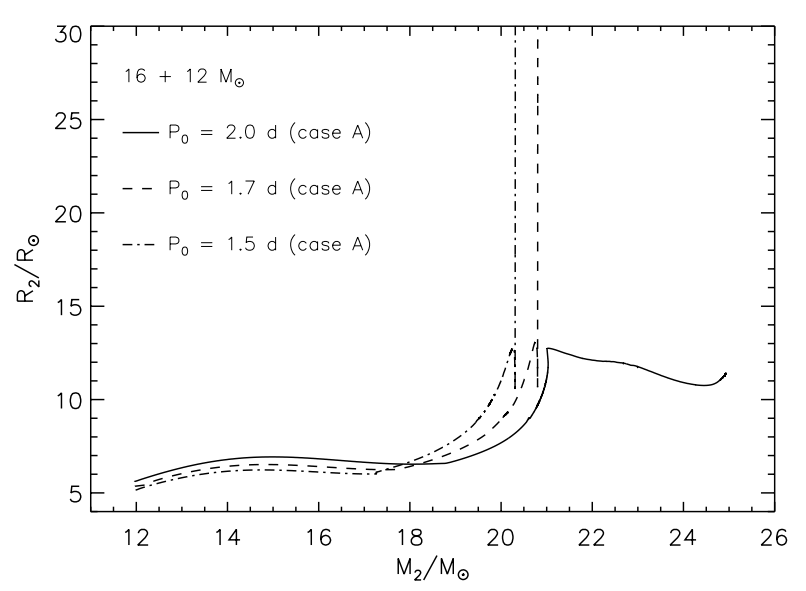

Fig. 7. Secondary radius as a function of its mass during Case A mass transfer, for three systems starting out with a $16 M_{\odot}$ and a $12 M_{\odot}$ component, with initial periods in the range 1.5...2 d (Systems Nos. 53...55). In the two closer systems, the secondaries terminate core hydrogen burning during the slow phase of the Case A mass transfer. Their ensuing rapid expansion leads to contact. In the $2 \mathrm{~d}$ period system No. 55 (solid line), Case $\mathrm{AB}$ mass transfer starts (at $M_{2} \simeq 21 M_{\odot}$ ) before the secondary has exhausted hydrogen in the core, and contact is avoided (see text)

radius evolution of three secondaries in Case A systems which have identical initial parameters except the period (systems Nos. 53...55 in Table 3). All three secondaries evolve through the rapid part of the Case A mass transfer (cf. Sect. 3.1) without expanding significantly (which is different for initial mass ratios $q \lesssim 0.65$; cf. Sect. 5.1). However, in the two closer systems, the mass transfer appears so early in the evolution of the primary that the now more massive secondary finishes core hydrogen burning first. It then starts to expand, but soon fills its Roche lobe and attempts a reverse Case B mass transfer (in systems Nos. 53 and 54). Thus, both stars now fill their Roche lobes and come into contact while the primary is still burning hydrogen in its core; we designate this as premature contact. The likely outcome of premature contact is a merger because the corresponding periods are too small for a successful common envelope ejection. This effect marks the lower period limit for contact free binaries.

In model No. 55, the primary ends core H-burning first. Therefore, Case AB mass transfer (cf. Sect. 3.1) sets in before the secondary expands strongly. This has two effects: it drives the two stars further apart - the period increases from $5.24 \mathrm{~d}$ at the beginning to $93 \mathrm{~d}$ at the end of Case $\mathrm{AB}$ mass transfer -, and it enhances the mixing of hydrogen into the core (cf. Braun \& Langer 1995) and thus delays the evolution of the secondary. I.e., if the initial period exceeds a critical value, Case AB mass transfer starts before the secondary expands and premature contact can be avoided. If the secondary star does not rejuvenate, reverse Case B mass transfer is avoided as well. Curiously, the secondary star in our Case A system No. 1 finishes core hydrogen burning just after the end of the Case A mass transfer and before the beginning of the Case $\mathrm{AB}$ mass 
transfer. However, the accretion due to the Case AB mass overflow leads to a shrinkage of its radius, and contact is avoided (see also Fig. 11 below).

The lower period limit for contact free binaries depends sensitively on the convection criterion. As mentioned in Sect. 2, we compute stellar models using the Ledoux criterion for convection and semiconvection with an efficiency parameter of $\alpha_{\mathrm{sc}}=0.01$. The semiconvective mixing efficiency - which is infinite in the case of the Schwarzschild criterion for convection - determines to a large extent whether an accreting core hydrogen burning star rejuvenates or not (Braun \& Langer 1995). If it fails, which is the more likely for less efficient semiconvection and the more advanced the star is in its evolution when it starts to accrete, it retains an unusual structure, with a smaller helium core mass and a larger hydrogen envelope mass compared to single stars. As such a structure keeps the star relatively compact during all the advanced burning stages - i.e., it avoids the red supergiant stage (Braun \& Langer 1995) - it will not fill its Roche lobe after core hydrogen burning. This is the situation in all our Case A systems which avoid premature contact and have initial mass ratios of $q \gtrsim 0.65$.

For models computed with the Schwarzschild criterion, the secondaries in Case A systems rejuvenate during or shortly after the rapid Case A mass transfer. This results in longer main sequence life times for the secondaries, and thus leads to less premature contact situations. I.e., the critical initial period for premature contact is shifted to smaller values. In this case, the secondaries all expand to red supergiants. Assuming the primary star is still present at that time, this is likely to initiate reverse Case B mass transfer. As the life time of a rejuvenating star is extended, the primaries have often finished their evolution by that time and the binary may even be disrupted (cf. Pols 1994). However, as the life time of a low mass helium star (i.e. the primary of such a system) can be comparable to the remaining hydrogen burning life time of the very massive secondary, reverse Case B mass transfer onto the low mass helium star may also occur. In this case, the helium star builds up a hydrogen envelope and swells to red giant dimensions. We designate the ensuing contact situation in Table 3 as reverse contact.

This scenario is illustrated by five of our Case A systems (Nos. 25, 48, 50, 59, and 72) which are computed using the Schwarzschild instead of the Ledoux criterion (marked " $c$ " in Table 3). Nos. 25 and 59, initially having a $12 M_{\odot}$ and a $16 M_{\odot}$ primary, a mass ratio of 0.67 and 0.69 , and a period of $2.5 \mathrm{~d}$ and $2.0 \mathrm{~d}$, respectively, avoid premature contact. However, they encounter reverse contact when the primaries have burnt $91 \%$ and $80 \%$ of their helium in the core. Both systems avoid contact altogether when they are computed with our standard convection physics, and evolve a reverse supernova order. I.e., an increase of the semiconvective mixing efficiency may reduce the number of systems with premature contact but increase the number with reverse contact. From system Nos. 25 we conclude that, were the Schwarzschild crite- rion correct, the majority of Case A binaries with $12 M_{\odot}$ primaries would evolve into reverse contact. Comparing our models Nos. 58 and 59, which have identical initial conditions but vary in the treatment of convection, shows that also in systems with $16 M_{\odot}$ primaries the lower period limit for contact free systems increases.

In the three other systems computed with the Schwarzschild criterion, systems Nos. 48, 50, and 72, the primaries explode as supernovae before the reverse mass transfer can occur. Two of these (Nos. 48 and 72), when computed with our standard convection physics, have a reverse supernova order (see systems Nos. 47 and 71 in Table 3), the other (No. 50) does not (cf. system No. 49 in Table 3).

Thus, the lower period limit for contact free evolution with the Schwarzschild criterion is expected between the lower period limit and the limit for reverse supernova order for Ledoux criterion and $\alpha_{\mathrm{sc}}=0.01$. The one early case $\mathrm{A}$ system containing a $25 M_{\odot}$ primary (No. 72 ) also shows no reverse mass transfer before the supernova explosion of the primary, despite being a system with reverse supernova order when computed with our standard convection physics. We conclude that for large masses, the amount of contact free case A systems does not change very much for different convection physics. On the contrary, at $12 M_{\odot}$ primary mass and below no contact free case A system are expected at all if the Schwarzschild criterion is used.

\subsection{Contact formation due to extreme mass ratios}

\subsubsection{Case B}

While the previous two subsections concerned contact formation due to large (delayed contact) or small (premature and reverse contact) initial periods, we investigate here contact formation due to large or small initial mass ratios.

Figure 8 shows the mass transfer rate as function of the transferred amount of mass for three Case B systems with identical initial primary mass $\left(12 M_{\odot}\right)$ and period (6 d), but different initial secondary masses. For smaller initial mass ratios $q_{\mathrm{i}}=M_{2, \mathrm{i}} / M_{1, \mathrm{i}}$, the mass transfer rates are somewhat larger in the early part of the mass transfer, since the orbit shrinks more rapidly for smaller $q_{\mathrm{i}}$. For conservative evolution (also ignoring stellar winds), the minimum separation depends on initial separation $d_{\mathrm{i}}$ and mass ratio $q_{\mathrm{i}}$ as $d_{\mathrm{min}}=d_{\mathrm{i}}\left(\frac{4 q_{\mathrm{i}}}{\left(q_{\mathrm{i}}+1\right)^{2}}\right)^{2}$. As, in a given system, period and orbital separation scale as $P^{2} \propto d^{3}$, this means that in systems with the same initial period, the primary star is, at the time when $P=P_{\min }$, squeezed into a smaller volume for smaller $q_{\mathrm{i}}$. This makes the mass transfer rate during the first phase of Case B mass transfer larger for smaller $q_{\mathrm{i}}$.

However, the maximum mass transfer rate, which is achieved after mass ratio reversal (cf. Sect. 4.1), is very similar in all three systems, even though the post-mass transfer periods $(115 \mathrm{~d}, 87 \mathrm{~d}$, and $72 \mathrm{~d}$ for secondary initial 


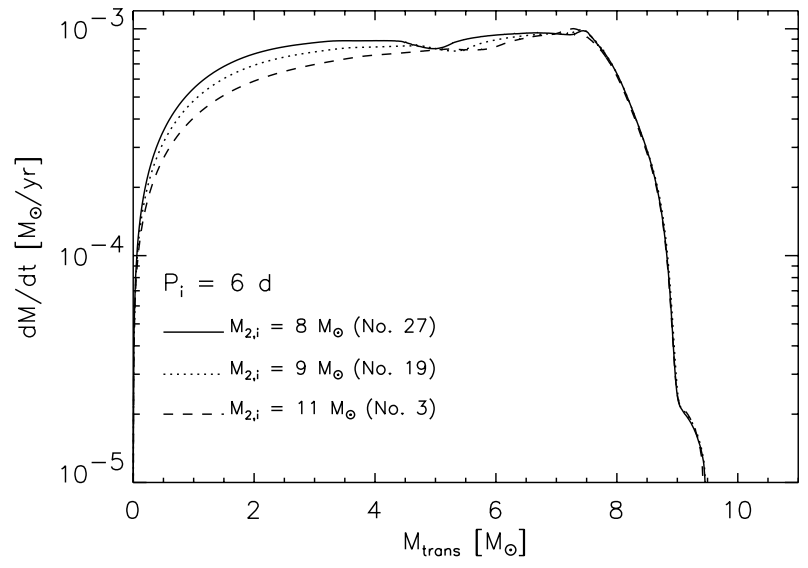

Fig. 8. Mass transfer rates as function of the transfered amount of mass for three Case B systems with $12 M_{\odot}$ primaries. The initial masses of the secondaries are $11 M_{\odot}$ (system No. 3), $9 M_{\odot}$ (No. 19), and $8 M_{\odot}$ (No. 27), as indicated. The initial period is $6 \mathrm{~d}$ for all three systems

masses of $11 M_{\odot}, 9 M_{\odot}$, and $8 M_{\odot}$, respectively) are not the same. Also the amount of mass which is transferred is independent of the secondary mass - it consists of the whole hydrogen-rich envelope of the primary.

Whether or not contact is reached is here mostly determined by the reaction of the secondary star to the accretion. Figure 9 shows that the primary with the lowest initial mass swells most during the mass transfer. The smallest initial mass ratio for which contact is avoided corresponds roughly to the condition that the mass accretion time scale of the secondary $M_{2} / \dot{M}_{2}$ remains smaller than its thermal time scale $G M_{2}^{2} /\left(R_{2} L_{2}\right)$. We designate contact due to this reason as $q$-contact.

As $q$-contact is established during the first phase of Case B mass transfer, it is quite distinct from delayed contact (Sect. 4.1). As $q$-contact evolves early during Case B mass transfer, it does not allow a major amount of mass to be accreted by the secondary. I.e., either the major part of the primary's hydrogen-rich envelope can be ejected from the system, or both stars merge. The situation that the secondary star can accrete significant amounts after a non-conservative early $q$-contact phase is unlikely since it would bring both stars even closer together.

In principle, there is also a maximum initial mass ratio for contact-free evolution of Case B systems: for $q_{\mathrm{i}}=1$, both stars age at the same rate and attempt to expand to red giants simultaneously. However, since the limiting value is very close to $q_{\mathrm{i}}=1$, we ignore this effect here as statistically insignificant.

\subsubsection{Case $A$}

The maximum mass transfer rate in Case A systems is achieved during the rapid mass transfer phase while the orbit is shrinking. Figure 10 shows the mass transfer rates of five Case A systems with identical initial conditions except for the initial secondary mass and thus the initial mass ratio $q_{\mathrm{i}}$. As shown in Sect. 4.2.1, the smaller $q_{\mathrm{i}}$ the

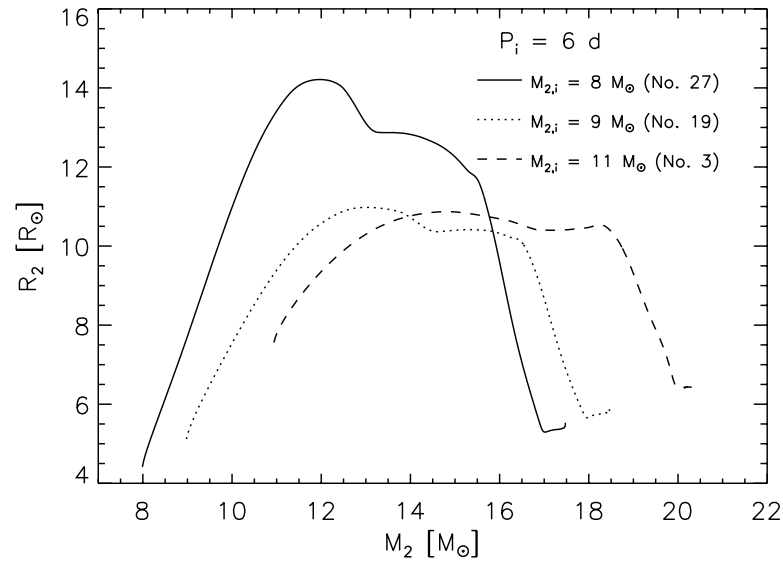

Fig. 9. Radii of the secondary stars as function of their mass for the same systems as shown in Fig. 8

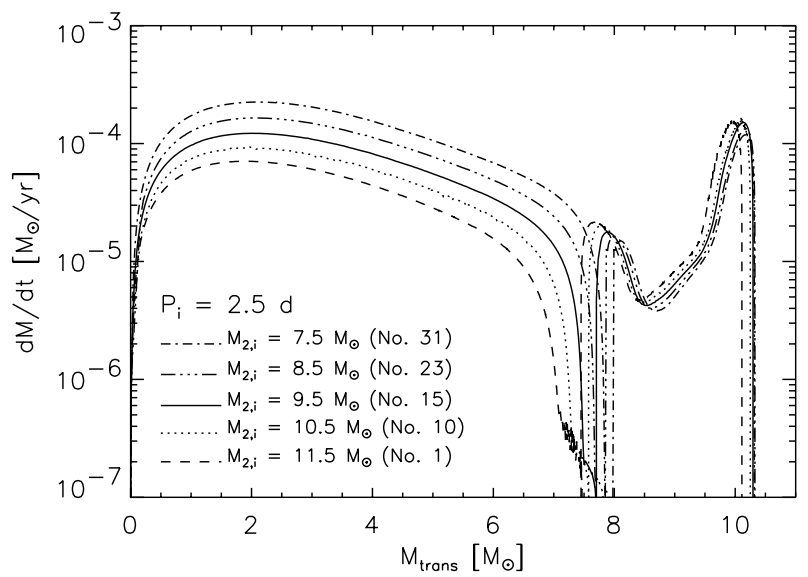

Fig. 10. Mass transfer rate as a function of transfered amount of mass, for five Case A systems with the same initial primary mass $\left(12 M_{\odot}\right)$ and initial period $(2.5 \mathrm{~d})$. The initial secondary masses are $11.5 M_{\odot}\left(\right.$ System No. 1), $10.5 M_{\odot}$ (No. 10), $9.5 M_{\odot}$ (No. 15), $8.5 M_{\odot}\left(\right.$ No. 23), and $7.5 M_{\odot}($ No. 31)

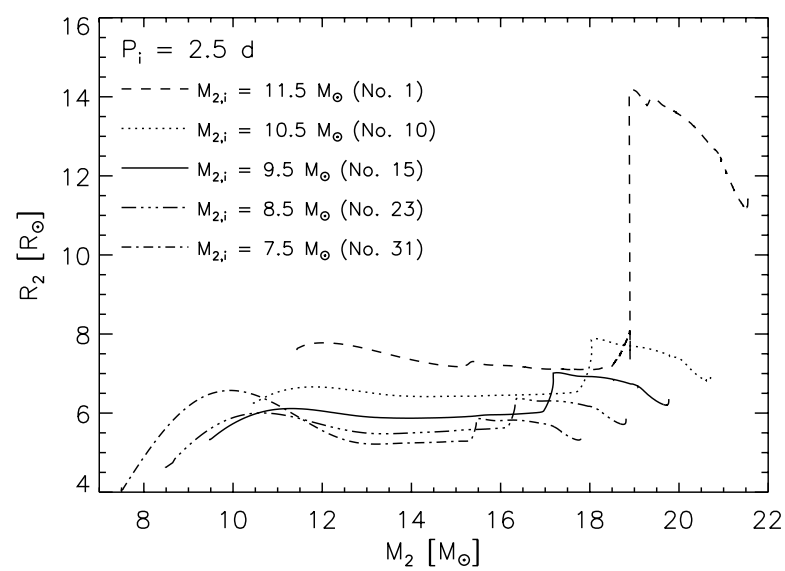

Fig. 11. Radii of the secondary stars as function of their mass for the same systems shown in Fig. 10. The first maximum is achieved during rapid Case A mass transfer. The second maximum is achieved during slow Case A mass transfer due to the nuclear evolution of the secondary. The radius decrease after the second maximum is due to the Case $\mathrm{AB}$ mass transfer 
larger is the maximum mass transfer rate since the primary is squeezed into a smaller volume. Also, more mass is transfered during the rapid Case A mass transfer for smaller $q_{\mathrm{i}}$. As for Case B, $q$-contact may occur for small $q_{\mathrm{i}}$ due to higher maximum mass transfer rates and due to larger thermal time scales of the secondary, the latter effect being the more important one. In Fig. 11, all systems shown avoid $q$-contact, but the relative radius increase during the rapid Case A mass transfer is clearly larger for the systems with smaller initial secondary masses.

The second expansion of the secondaries during the Case A mass transfer (Fig. 11) is due to their nuclear evolution during the slow mass transfer phase. It is ended by the onset of Case AB mass transfer. The secondary star in system No. 1 has already ended core hydrogen burning and expanded appreciably before the onset of Case AB mass transfer. The onset of Case AB mass transfer stops its expansion and prevents the system from evolving into premature contact. However, Figs. 10 and 11 demonstrate, that for too large mass ratios, massive Case A binaries evolve into premature contact as in the case of too small initial periods (cf. Sect. 4.1.2). I.e., while Case B systems evolve into contact only when their initial mass ratio is too large, Case A binaries can do so for too large or too small initial mass ratio.

Figure 10 shows also, that the total amount of mass which is transfered during case $\mathrm{A}$ and $\mathrm{AB}$ is nearly independent of $q_{\mathrm{i}}$. This can not be expected in general, as we shall see below that the primary mass after Case A and AB mass transfer depends on the core hydrogen abundance at the onset of mass transfer and on the initial mass ratio (cf. Fig. 14 below). While smaller initial mass ratios lead to smaller primary masses, the larger Roche lobe due to the lower secondary mass (for the same period) leads to mass transfer only later in the evolution of the primary and thus to larger post-mass transfer primary masses. Both effects cancel each other in the models shown in Fig. 10.

\section{Discussion}

\subsection{The contact-free regime}

Figures 12 and 13 summarise the range of contact-free evolution for systems with $12 M_{\odot}$ and $16 M_{\odot}$ primaries, respectively. For all mass transfer cases and primary masses, there is a rather well defined critical initial mass ratio $q_{\mathrm{c}}$ such that only systems with larger $q_{\text {i }}$ avoid contact. For $q_{\mathrm{i}}<q_{\mathrm{c}}$, they evolve into $q$-contact. Likely, most of the systems undergoing $q$-contact merge, since contact occurs early during the mass transfer, at a small orbital separation. We find $q_{\mathrm{c}} \simeq 0.65$ for all Case $\mathrm{A}$ systems with primaries in the initial mass range $12 \ldots 25 M_{\odot}$. For Case B systems, $q_{\mathrm{c}}$ decreases from $q_{\mathrm{c}} \simeq 0.7$ at $12 M_{\odot}$ to $q_{\mathrm{c}} \simeq 0.8$ at $16 M_{\odot}$ and $q_{\mathrm{c}} \simeq 1$ at $25 M_{\odot}$ initial primary mass.

The range of contact-free Case A systems is further limited by the possibility of premature contact, which occurs for initial periods below a certain threshold, but also for initial mass ratios above a critical value, i.e. too close

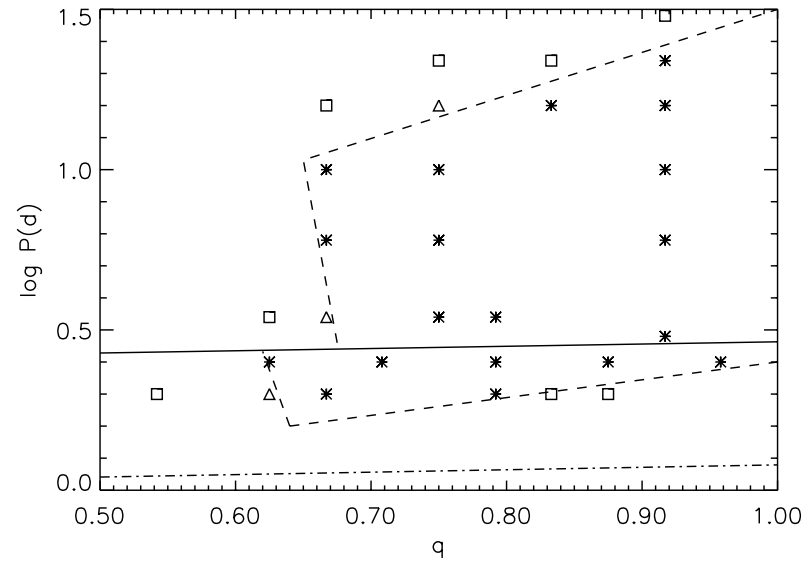

Fig. 12. Distribution of all computed binaries with $12 M_{\odot}$ primaries in the $\log P$ versus $q$-diagram. Asterisks mark contactfree systems, while squares mark systems which evolve into contact. Systems marked with triangles are borderline cases, i.e., they evolve into a short contact phase but the secondary radius never exceeds its Roche radius by more than a factor 1.5. The solid line separates Case A (below) and Case B systems. All case A systems for this primary mass have a reverse supernova order. The dashed lines indicate the boundary between contact-free and contact evolution. The dashed-dotted line is defined by the condition that the primary fills its Roche lobe already on the zero age main sequence

to one. We should note that for even smaller initial periods than investigated here, premature contact may occur already before the secondary has finished core hydrogen burning (cf. Pols 1994). In any case, the outcome of premature contact is most likely the merging of both stars. The possibility of reverse contact and of a reverse supernova order for the Case A systems, and their dependence on the semiconvective mixing time scale are discussed in the next section.

As described in Sect. 4.1.2, the uncertain efficiency of semiconvective mixing introduces a considerable uncertainty to the evolution of Case A systems. Semiconvective mixing affects the rejuvenation process in an essential way, as it is this process which transports hydrogen into the convective core of the accretion stars. While for infinitely fast semiconvective mixing - which corresponds to the use of the Schwarzschild criterion for convection - all accretion stars rejuvenate (Hellings 1983), this is not the case for finite semiconvective mixing time scales (Braun \& Langer 1995). Longer mixing time scales lead to shorter core hydrogen burning life times of the secondaries. Therefore, slow semiconvection - as assumed here - leads to earlier contact. Nevertheless, slow semiconvection leads perhaps not to a smaller total number of contact systems, since for those systems which avoid premature contact, the chance of reverse contact during core helium burning of the secondary is much smaller than in the case of fast semiconvective mixing (cf. Sect. 4.1.2).

We note that although semiconvection can be important for the upper end of the mass spectrum of our study (cf. also Wellstein \& Langer 1999), another effect 


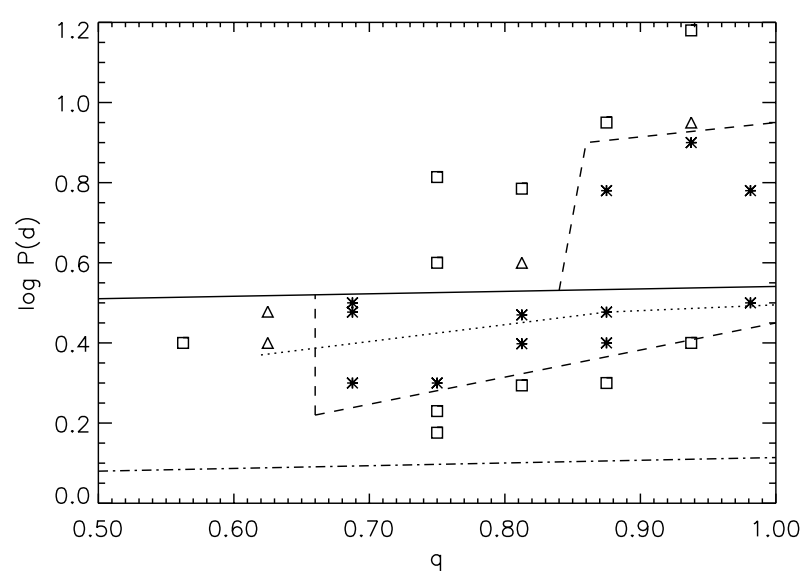

Fig. 13. The same as in Fig. 12 but for systems with initial primary masses of $16 M_{\odot}$. The dotted line divides the contact-free Case A systems into such with normal and reverse supernova order, the latter occurring in the systems below the line

dominates for the less massive Case A systems. In our systems with an initial primary mass of $12 M_{\odot}$, the luminosity ratio after the mass transfer is very large, of the order of 100, while it is only of the order of 10 for our most massive systems (see Table 3 ). The reason for the drop of the luminosity ratio for higher primary masses is simply the flattening of the stellar mass-luminosity relation for increasing mass. As the luminosity ratio reflects the ratio of the evolutionary speeds of both components, it is clear that with a post-mass transfer value of 100, only very late Case A systems can avoid contact - independent of whether the secondary rejuvenates or not. The large luminosity ratio for lower primary initial masses is also the main reason why the initial parameter space for contactfree evolution becomes smaller for lower mass systems.

Finally, there is an upper period which limits the contact-free regime of the Case B systems. Our calculations have shown that systems close to this limit may experience delayed contact, with the consequence of considerable accretion on the secondary, and a high chance to avoid the merging of both stars. Initially even wider systems may correspond more to the standard common envelope scenario, again with the possibility to avoid a merger, but likely without significant accretion of the secondary.

By comparing Figs. 12 and 13, and including our results for even more massive systems (see Table 3, and Wellstein \& Langer 1999), it becomes clear that the range of contact-free Case A systems becomes smaller for smaller initial primary masses, while that for contact-free Case B systems becomes smaller for larger ones. E.g., there are no contact-free Case B systems for initial primary masses of $25 M_{\odot}$. We conclude that at lower masses, conservative evolution is to be expected mainly from close Case B systems, while at larger masses Case A systems dominate the conservative evolution. Considering that massive binaries with initial periods of up to some thousand days

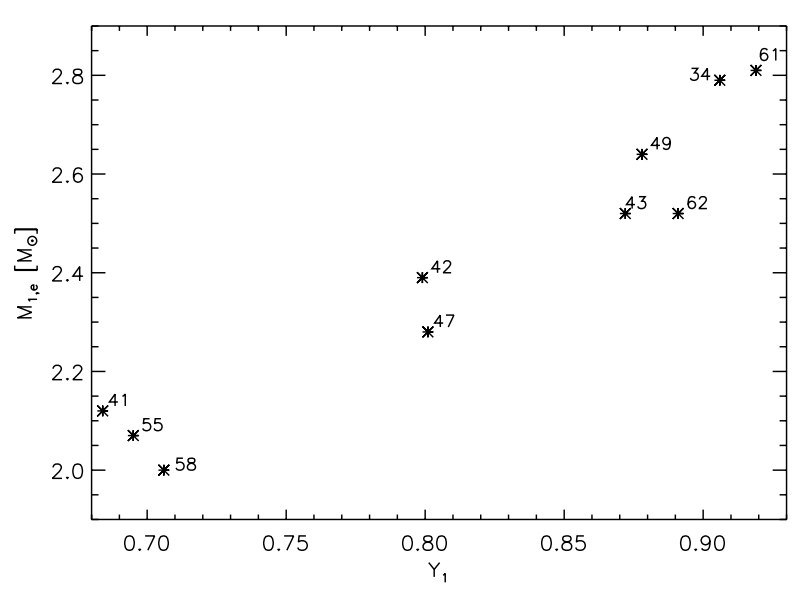

Fig. 14. Masses after Case AB mass transfer for primaries with an initial mass of $16 M_{\odot}$, as function of their central helium abundance at the onset of Case A mass transfer. For comparison, the post Case B mass transfer masses of $16 M_{\odot}$ primaries are all about $3.6 M_{\odot}$

may interact, and adopting an equal number of binaries per $\log P$ bin, up to one third of all interacting systems in the considered mass range and with $q_{\mathrm{i}} \gtrsim 0.7$ may evolve conservatively.

\subsection{Remnant types}

Those of our systems which avoid merging evolve into $\mathrm{OB}+\mathrm{He}$ star systems. We discuss in the following the further evolution of the primaries, the secondaries, and of merger stars.

\subsubsection{Primaries}

Table 3 shows that the helium star masses after the Case $A B / B$ mass transfer in our systems fall into the range $1 . . .7 M_{\odot}$. The helium star masses in the Case $\mathrm{B}$ systems correspond simply to the helium core mass of the primary at core hydrogen exhaustion, with a very small scatter $\left(+0.1 M_{\odot}\right)$. Figure 14 shows that the situation is more complex for the Case A systems. We find a strong dependence of the helium star mass on the initial period, i.e. on the central hydrogen abundance of the primary when the mass transfer begins. We also find a weaker dependence on the initial mass ratio (cf., systems Nos. 41, $55,58)$. Figure 14 demonstrates that our $16 M_{\odot}$ Case A primaries become $2 \ldots 3 M_{\odot}$ helium stars - compared to a Case B post-mass transfer mass of $\sim 3.6 M_{\odot}$, which is independent of other parameters. We point out that these strongly reduced Case A primary masses are often ignored in simplified binary studies which rely on single star evolutionary models.

The further evolution of the helium stars depends strongly on their mass, and partly on the evolution of their companion stars. The more massive helium stars in our mass range correspond to Wolf-Rayet stars, and will 
undergo wind mass loss before they explode as types Ib/Ic supernova. Those helium stars with masses below $\sim 4 M_{\odot}$ expand to giant dimensions after core helium exhaustion. Depending on whether the companion star is still present at that time, this may lead to Case $\mathrm{ABB} / \mathrm{BB}$ mass transfer, and a further reduction of the mass of the helium star by $\sim 0.5 M_{\odot}$ or so (cf. system No. 37 in Sect. 3.2). Independent of this, the helium stars with final masses in the range $2 \ldots 4 M_{\odot}$ are likely to explode as helium $g i$ ants. The effect of the large radii of these stars on the supernova light curve is still unexplored. Helium stars in the mass range $1.4 \ldots 2 M_{\odot}$ could, in principle, also make it to core collapse, but as their post-core helium burning life time is rather large it may be more likely that stellar winds will reduce their mass below the Chandrasekhar mass and they end up as white dwarfs, like their counterparts with masses between $1 \ldots 1.4 M_{\odot}$.

We point out that all $12 M_{\odot}$ primaries in our contactfree Case A systems end up as white dwarfs, and even some $16 M_{\odot}$ primaries may do so (e.g., system No. 55). I.e., the limiting initial mass separating white dwarf formation from core collapse is largely increased in Case A binaries, compared to single stars. We note that this effect exists also for Case B binaries, strongly so for primaries which undergo a Case BB mass transfer, but at a moderate level even otherwise. The same effect for the limiting initial mass between neutron star and black hole formation has been discussed by Wellstein \& Langer (1999).

Finally, we can not exclude that some of the helium stars with masses close to but above the Chandrasekhar mass develop a degenerate $\mathrm{C} / \mathrm{O}$-core and ignite carbon explosively. In that case, they would produce a type Ia supernova. However, in the $1.45 M_{\odot}$ helium star of system No. 31 (cf. Sect. 3.1), a luminosity of $\sim 10^{4} L_{\odot}$ during shell helium burning implies a growth rate of the $\mathrm{C} / \mathrm{O}$ core mass of $\sim 10^{-6} M_{\odot} \mathrm{yr}^{-1}$, which is fast enough to allow carbon to ignite non-explosively.

\subsubsection{Secondaries}

All our secondary stars evolve to core collapse. In Case A systems, about half of the secondaries does so before the primary has ended its evolution (cf. Table 3). As most of our primaries did not rejuvenate, they remain blue supergiants throughout their post-main sequence evolution (cf. Figs. 1 and 3). When they explode as type II supernova, they will thus resemble SN 1987A. We noted in Sect. 3.1 that the secondary of system No. 31 provides a viable progenitor model for SN 1987A. In this case, there should be a $\sim 1.5 M_{\odot}$ helium star left in the supernova remnant. In similar systems, however, the supernova order is not reversed and no such helium star would be expected (cf. Table 3).

\subsubsection{Merger}

Several of our systems evolve into contact and are likely to lead to a merger. While it is beyond our scope to predict the properties of the merged stars, we can distinguish two types of events. If the merging occurs during Case A mass transfer and before the secondary has terminated core hydrogen burning, the merged object will still be a core hydrogen burning star. Its surface may be enriched in hydrogen burning products, but otherwise it might resemble a normal main sequence star in many respects.

However, a merger during a Case B or reverse Case B mass transfer (cf. Sect. 4.1.1; note that the latter may even occur during a Case A mass transfer; cf. Fig. 7) has a different result. Since the original primary has already developed a compact hydrogen-free core, the merger star will have a hydrogen-free core with a mass close to that of the primary. It is uncertain how much of the hydrogen-rich material is lost during the merging process, and whether some hydrogen-rich matter can penetrate into the helium core. However, if the mass of the merger star exceeds the mass of the primary star, which appears likely, then the helium core mass of the merger star is much smaller than the helium core mass of a single star of comparable mass. I.e., its structure is similar to that of our non-rejuvenating accretion stars during core helium burning. As a consequence of their small helium core mass, these stars may avoid to evolve into red supergiants (Podsiadlowski et al. 1990, 1992).

\subsubsection{Reverse supernova order}

In our Case A binaries, a reversal of the supernova order appears in all systems containing a $12 M_{\odot}$ primary (cf. Fig. 12) and in some systems containing a $16 M_{\odot}$ primary (cf. Fig. 13). At $12 M_{\odot}$, supernova order reversal depends not so much on whether or not the secondary star rejuvenates. Instead, the mass ratio after the rapid phase of the Case A transfer, and thereby the luminosity ratio, is essential. Due to the strong decrease of the primaries mass, it becomes a factor of about 100 less luminous than the secondary. Therefore, independent of how much fresh hydrogen is mixed into the secondaries core, it finishes its evolution first as long as no reverse mass transfer occurs.

However, in case of rapid semiconvective mixing, the secondary may rejuvenate, with the consequence of reverse mass transfer before it can evolve into a supernova. Due to the extreme mass ratio, the reverse mass transfer is expected to be non-conservative. Its outcome is either a merger or a short period double helium star binary. Assuming the latter, we can estimate whether or not the supernova order would be reversed. In system No. $17-$ computed with $\alpha_{\mathrm{sc}}=0.02$ - the secondary is more evolved at time of reverse mass transfer, i.e. both stars burn helium in their cores, with central helium mass fractions of $0.5 / 0.3$ for the primary/secondary. Thus the supernova order is expected to be reversed. For system No. 25 computed with Schwarzschild criterion, it is less clear what 
happens. The primary has already spent about $610^{6} \mathrm{yr}$ at central helium burning and has reached a core helium mass fraction of 0.09 . The secondary has reached a core helium mass fraction of 0.63 after only $310^{5}$ years of central helium burning. Because the primary's helium shell burning phase is expected to last more than $110^{6}$ years (cf. system No. 31 in Table 1) it appears possible that also in this system the supernova order is reversed, if the system does not merge.

For systems with an initial primary mass of $25 M_{\odot}$ the convection physic dominates the question whether the supernova order is reversed. This can be seen in systems Nos. 65, 66 and 67, where the value for the efficiency parameter for semiconvective mixing $\alpha_{\mathrm{sc}}$ is the only difference. Only in system No. 65 with $\alpha_{\mathrm{sc}}=0.01$ the supernova oder is reversed. With $\alpha_{\text {sc }} \geq 0.02$ no reversal appears. Also the case B system 68 reverses its supernova order with $\alpha_{\mathrm{sc}}=0.01$. This shows that the mass of the primary after mass transfer in this systems is not very important because the nuclear time scales of the stars in this mass range vary less with mass than for lower mass stars. Also the early case A binary No. 71 has a reverse supernova order in contrast to system No. 72, which has the same parameters, but is calculated with the Schwarzschild criterion. The systems with $16 M_{\odot}$ primary are an intermediate case, but seems to behave more like the systems containing a $25 M_{\odot}$ primary (cf. systems Nos. 47 and 48 ).

Whether or not a reverse mass transfer occurs before the primary becomes a compact object depends strongly on the semiconvective mixing speed. For fast semiconvective mixing, the secondaries become red supergiants. Then, the crucial question is not if reverse mass transfer occurs but when it occurs. A shorter semiconvective mixing time scale has two effects. First, the maximum radius of the secondary after core hydrogen exhaustion increases (cf. Wellstein \& Langer 1999). This makes it more likely for the secondary to fill its Roche lobe and transfer matter back to the primary. Second, the time the secondary spends on the main sequence increases, as more hydrogen is mixed into the convective core during central hydrogen burning. Thus it is less likely that the reverse mass transfer occurs before the primary has finished its evolution and has become a compact object.

Although in general, systems which have a normal supernova order would be able to avoid reverse mass transfer and vice versa, some systems which show a reverse supernova order for slow semiconvective mixing $\left(\alpha_{\mathrm{sc}}=0.01\right)$ avoid reverse mass transfer during the lifetime of the primary when the Schwarzschild criterion is used, as shown by systems Nos. 48 and 72 .

\subsection{Comparison to previous work}

Pols (1994) presented 24 binary models for initial primary masses in the range $8 \ldots 16 M_{\odot}$, mostly for Case A systems. Since he used the Schwarzschild criterion for convection, the secondary components rejuvenated in all com- puted cases. As a consequence, contact phases during slow case A mass transfer occurred for shorter periods than in our models. Furthermore, due to the rejuvenation, a supernova explosion of the secondary as the first star could only appear after a reverse mass transfer phase. This mass transfer appears at very large mass ratios and must therefore be non-conservative. As discussed by Pols, it is an open question whether or not such a binary merges or is able to expel the secondary's hydrogen-rich envelope to form a close binary consisting of two helium stars. As the helium star resulting from the secondary is then the more massive component, a supernova order reversal is possible if the merging of both stars can be avoided.

Sybesma (1985) computed 5 binary sequences starting with a primary mass of $20 M_{\odot}$, a secondary mass of $10 M_{\odot}$, and periods ranging from 1.5 up to 10 days. His results differ from ours mainly due his assumption of huge convective core overshooting. This leads to much more massive helium-rich cores in the main sequence models. Consequently, he obtains Case A mass transfer for initial periods which fall in our Case B regime. As Case A mass transfer is more likely conservative in this mass range, Sybesma obtains conservative evolution for larger periods than we (e.g. 10 days), and for smaller initial mass ratio $(q=0.5)$.

Several authors specify the fraction $\beta$ of the primary's envelope which is accreted by the secondary in advance. E.g., based on observations, De Greve \& de Loore (1992) and de Loore \& De Greve (1992) use $\beta=0.5$, which means half of the primary's envelope mass leaves the system, together with an amount of angular momentum which needs to be specified additionally. While an average value of $\beta=0.5$ appears in fact possible, our models favour the idea that $\beta$ decreases continuously for larger initial periods, with $\beta=1$ for systems which avoid contact and $\beta=0$ for large initial periods. E.g., we obtained $\beta=0.68$ and $\beta=0.60$ for our systems Nos. 7 and 8 (cf. Table 3). By comparison with De Greve \& de Loore (1992) and de Loore $\&$ De Greve (1992), we note that the contact-free regime is expanded somewhat if $\beta=0.5$ is used ab initio.

\section{Comparison with observations}

While it is unlikely that any of the binary systems considered here has an observable counterpart during the mass transfer - with the exception of the slow phase of the Case A mass transfer - they have a relatively long-lived post-mass transfer stage. We emphasise that this is true for those binary which survive the mass transfer, but also for those which lead to mergers. Therefore, we characterise the typical properties of systems after the first mass transfer phase in Table 4. At this stage, the systems consist of a main sequence or supergiant $\mathrm{O}$ or early $\mathrm{B}$ star of $18 \ldots 40 M_{\odot}$, and a helium star in the mass range $1 \ldots 7 M_{\odot}$. The orbital periods range from $24 \mathrm{~d}$ to $255 \mathrm{~d}$.

An inspection of Table 4 indicates that it may be a challenging task to try to detect systems like these observationally. A photometric investigation will have limited 
Table 4. Observable quantities for our contact-free systems. After system number, initial values of primary mass $M_{1}$, secondary mass $M_{2}$, and period $P_{i}$, we give quantities obtained after the Case AB or Case B mass transfer, at a time when the core helium mass fraction of the primary has decreased to 0.8 during core helium burning: primary and secondary mass and mass ratio, bolometric luminosities, effective temperatures (where no wind effects are included for the helium stars), stellar radii, the radius of the secondary in units of its Roche radius, orbital separation, system period, and orbital velocities. As in Table 3, bold values for the initial masses identify the component which ends its evolution first, typically by a supernova explosion. Both components of system No. 43 explode simultaneously, within the accuracy of the computation

\begin{tabular}{|c|c|c|c|c|c|c|c|c|c|c|c|c|c|c|c|c|c|}
\hline No. & $M_{1}$ & & $\begin{array}{l}P_{i} \\
\mathrm{~d}\end{array}$ & $\begin{array}{r}M_{1}^{\prime} \\
\quad N\end{array}$ & $M_{2}^{\prime}$ & $q^{\prime}$ & $\begin{array}{l}L_{1}^{\prime} \\
\log \end{array}$ & $\begin{array}{l}L_{2}^{\prime} \\
\odot)\end{array}$ & $T_{\mathrm{eff}, 1}^{\prime}$ & $T_{\mathrm{eff}, 2}^{\prime}$ & $R_{1}^{\prime}$ & $R_{2}^{\prime}$ & $\begin{array}{c}R_{2}^{\prime} \\
R_{\text {Roche }}\end{array}$ & $\begin{array}{c}a^{\prime} \\
R_{\odot} \\
\end{array}$ & $\begin{array}{r}P^{\prime} \\
\mathrm{d}\end{array}$ & & $\begin{array}{c}v_{2}^{\prime} \\
\mathrm{s}^{-1} \\
\end{array}$ \\
\hline 1 & 12 & 11.5 & 2.5 & 1.42 & 21.5 & 15.1 & 2.98 & 4.99 & 56 & 25 & 0.3 & 16.5 & 0.06 & 433 & 218 & 94 & 6.2 \\
\hline 2 & 12 & 11 & 3 & 2.44 & 20.4 & 8.4 & 3.93 & 4.74 & 63 & 33 & 0.8 & 7.2 & 0.07 & 170 & 54 & 143 & 17.1 \\
\hline 3 & 12 & 11 & 6 & 2.38 & 20.4 & 8.6 & 3.82 & 4.79 & 65 & 35 & 0.6 & 6.8 & 0.04 & 282 & 115 & 111 & 13.0 \\
\hline 10 & 12 & 10.5 & 2.5 & 1.42 & 20.7 & 14.6 & 2.97 & 4.87 & 56 & 34 & 0.3 & 7.9 & 0.03 & 390 & 190 & 97 & 6.7 \\
\hline 12 & 12 & 10 & 16 & 2.43 & 19.4 & 8.0 & 3.81 & 4.71 & 64 & 34 & 0.6 & 6.6 & 0.02 & 472 & 255 & 83 & 10.4 \\
\hline 15 & 12 & 9.5 & 2.5 & 1.41 & 19.7 & 14.0 & 2.96 & 4.79 & 56 & 33 & 0.3 & 7.5 & 0.03 & 351 & 166 & 100 & 7.1 \\
\hline 16 & 12 & 9.5 & 3.5 & 2.45 & 18.9 & 7.7 & 3.94 & 4.63 & 62 & 33 & 0.8 & 6.3 & 0.07 & 158 & 50 & 142 & 18.4 \\
\hline 18 & 12 & 9 & 3.5 & 2.32 & 18.5 & 8.0 & 3.74 & 4.63 & 66 & 33 & 0.6 & 6.5 & 0.07 & 163 & 53 & 138 & 17.4 \\
\hline 19 & 12 & 9 & 6 & 2.36 & 18.5 & 7.8 & 3.81 & 4.63 & 65 & 33 & 0.6 & 6.2 & 0.04 & 227 & 87 & 117 & 15.0 \\
\hline 23 & 12 & 8.5 & 2.5 & 1.43 & 18.8 & 13.1 & 2.97 & 4.72 & 57 & 33 & 0.3 & 6.8 & 0.04 & 301 & 135 & 105 & 8.0 \\
\hline 24 & 12 & 8 & 2 & 1.12 & 18.5 & 16.5 & 2.64 & 4.84 & 48 & 32 & 0.3 & 8.5 & 0.04 & 373 & 189 & 94 & 5.7 \\
\hline 27 & 12 & 8 & 6 & 2.37 & 17.5 & 7.4 & 3.79 & 4.55 & 65 & 33 & 0.6 & 5.8 & 0.05 & 197 & 72 & 122 & 16.5 \\
\hline 31 & 12 & 7.5 & 2.5 & 1.45 & 17.8 & 12.3 & 2.99 & 4.64 & 57 & 33 & 0.3 & 6.3 & 0.04 & 249 & 104 & 112 & 9.1 \\
\hline 34 & 16 & 15.7 & 3.2 & 2.79 & 27.8 & 10.0 & 3.88 & 5.14 & 76 & 37 & 0.5 & 9.3 & 0.07 & 234 & 75 & 143 & 14.4 \\
\hline 35 & 16 & 15.7 & 6 & 3.49 & 27.5 & 7.9 & 4.17 & 5.14 & 80 & 39 & 0.6 & 8.3 & 0.06 & 257 & 86 & 134 & 17.0 \\
\hline 37 & 16 & 15 & 8 & 3.63 & 26.7 & 7.4 & 4.20 & 5.10 & 80 & 36 & 0.7 & 8.9 & 0.06 & 275 & 96 & 128 & 17.3 \\
\hline 38 & 16 & 15 & 9 & 3.64 & 26.7 & 7.3 & 4.22 & 5.10 & 80 & 38 & 0.7 & 8.1 & 0.05 & 295 & 107 & 123 & 16.8 \\
\hline 42 & 16 & 14 & 2.5 & 2.39 & 26.5 & 11.1 & 3.78 & 5.17 & 68 & 34 & 0.6 & 11.0 & 0.07 & 274 & 98 & 130 & 11.7 \\
\hline 43 & 16 & 14 & 3 & 2.52 & 26.6 & 10.6 & 3.75 & 5.10 & 72 & 36 & 0.5 & 9.0 & 0.06 & 280 & 101 & 128 & 12.2 \\
\hline 44 & 16 & 14 & 6 & 3.60 & 25.8 & 7.2 & 4.19 & 5.04 & 80 & 38 & 0.6 & 7.8 & 0.07 & 214 & 67 & 142 & 19.8 \\
\hline 47 & 16 & 13 & 2.5 & 2.28 & 26.1 & 11.4 & 3.65 & 5.11 & 66 & 36 & 0.5 & 9.1 & 0.06 & 274 & 99 & 129 & 11.3 \\
\hline 49 & 16 & 13 & 3 & 2.64 & 25.3 & 9.6 & 3.84 & 5.07 & 74 & 35 & 0.5 & 9.1 & 0.08 & 204 & 64 & 146 & 15.2 \\
\hline 51 & 16 & 13 & 4 & 3.55 & 24.8 & 7.0 & 4.19 & 4.99 & 80 & 36 & 0.7 & 8.2 & 0.10 & 152 & 41 & 164 & 23.6 \\
\hline 55 & 16 & 12 & 2 & 2.07 & 24.8 & 12.0 & 3.57 & 5.17 & 62 & 33 & 0.5 & 11.8 & 0.08 & 258 & 93 & 130 & 10.8 \\
\hline 56 & 16 & 12 & 4 & 3.55 & 23.9 & 6.7 & 4.17 & 4.93 & 80 & 37 & 0.6 & 7.0 & 0.09 & 141 & 37 & 168 & 24.9 \\
\hline 58 & 16 & 11 & 2 & 2.00 & 24.1 & 12.1 & 3.56 & 5.10 & 62 & 33 & 0.5 & 10.8 & 0.07 & 249 & 89 & 131 & 10.8 \\
\hline 60 & 16 & 11 & 3 & 2.52 & 23.8 & 9.4 & 3.74 & 4.95 & 73 & 37 & 0.5 & 7.3 & 0.07 & 194 & 61 & 145 & 15.4 \\
\hline 61 & 16 & 11 & 3.2 & 2.81 & 23.3 & 8.3 & 3.87 & 4.93 & 77 & 36 & 0.5 & 7.4 & 0.09 & 151 & 42 & 162 & 19.5 \\
\hline 62 & 16 & 10 & 2.5 & 2.28 & 23.1 & 10.1 & 3.77 & 4.97 & 67 & 36 & 0.6 & 7.8 & 0.06 & 200 & 65 & 142 & 14.0 \\
\hline 65 & 25 & 24 & 3.5 & 5.15 & 40.6 & 7.9 & 4.53 & 5.54 & 98 & 38 & 0.6 & 13.7 & 0.14 & 179 & 41 & 196 & 24.8 \\
\hline 68 & 25 & 24 & 5 & 7.28 & 39.2 & 5.4 & 4.85 & 5.45 & 106 & 39 & 0.8 & 11.9 & 0.15 & 149 & 31 & 205 & 38.1 \\
\hline 70 & 25 & 23 & 4 & 5.31 & 39.4 & 7.4 & 4.56 & 5.49 & 99 & 39 & 0.6 & 12.4 & 0.13 & 172 & 39 & 196 & 26.4 \\
\hline 71 & 25 & 22 & 2.5 & 4.80 & 39.6 & 8.3 & 4.45 & 5.55 & 91 & 37 & 0.7 & 14.2 & 0.15 & 171 & 39 & 198 & 24.0 \\
\hline 73 & 25 & 19 & 4 & 5.26 & 35.9 & 6.8 & 4.55 & 5.38 & 99 & 43 & 0.7 & 8.7 & 0.11 & 140 & 30 & 206 & 30.2 \\
\hline 74 & 25 & 16 & 4 & 5.22 & 33.3 & 6.4 & 4.54 & 5.29 & 99 & 41 & 0.6 & 8.8 & 0.14 & 118 & 24 & 215 & 33.8 \\
\hline
\end{tabular}

success due to three factors. First, the luminosity ratio of both stars is large, mostly of the order of 10 , with the worst case of 160 (system No. 24) and the best of 4 (sys- tem No. 68). Second, the less luminous star has a much smaller radius than the brighter star, which minimises the brightness amplitude in case of an eclipse. Third, both 
stars underfill their Roche volumes by a large factor, which means that they are likely to radiate isotropically, and that eclipses are not likely.

We note that after core helium burning, all the helium stars below $\sim 4 M_{\odot}$ expand to giant dimensions and - if the OB star has not yet turned into a supernova may even fill their Roche volumes (cf. Sect. 3.2). As also their luminosities increase by up to a factor 10, a photometric detection may indeed be more likely in this stage. However, for the more massive helium stars $\left(M_{1}^{\prime} \gtrsim 3 M_{\odot}\right)$, this stage is very short lived $(\lesssim 0.1 \mathrm{Myr})$ compared to their core helium burning life times ( $\sim 1 \mathrm{Myr}$; cf. Table 2$)$. In systems with lower mass helium stars, the time scales are more favourable: the $1.45 M_{\odot}$ helium star of system No. 31 burns helium in its core for about $5 \mathrm{Myr}$, and its ensuing shell burning stage lasts at least $1 \mathrm{Myr}$, possibly more depending on the mass loss rate during this stage (cf. Sect. 3.1 and Table 1).

Table 4 also shows the orbital velocities of both components after the mass transfer. While the helium stars have favourably large velocities of the order of $100 \ldots 200 \mathrm{~km} \mathrm{~s}^{-1}$, they might be unobservable against the glare of their OB companion. The latter moves with only $5 \ldots 35 \mathrm{~km} \mathrm{~s}^{-1}$. While these velocities are small, it may not be hopeless to try to detect these motions in OB stars.

It is interesting to note that the assumption of conservative evolution leads, in all respects, to system properties which are less favourable for an observational detection of such systems, compared to the assumption of mass- and angular momentum loss from the system. This is demonstrated by the estimated outcome of our delayed contact system No. 7 in Sect. 4.1.1, which results in a shorter orbital period $(7 \mathrm{~d})$, correspondingly larger orbital velocities, and a mass- and luminosity ratio closer to 1 , compared to similar conservative systems. This tendency can also be verified in the results obtained by de Loore \& De Greve (1992) obtained with $\beta=0.5$, i.e. assuming that half of the transferred mass leaves the system.

The best chances to find post-mass transfer systems like those of Table 4 seems to be at the upper end of the considered mass range. Our systems with initial primary masses of $25 M_{\odot}$ obtain the smallest luminosity ratio $L_{2}^{\prime} / L_{1}^{\prime}$, the shortest periods, and the largest orbital velocities. The helium star masses in these systems are in the range $5 \ldots 7 M_{\odot}$, which makes the stars comparable to Wolf-Rayet stars, or, more specifically, WN stars. However, although we know $12 \mathrm{OB}+\mathrm{WN}$ systems with established mass ratios and orbital periods (van der Hucht 2000 ), none of them fits to our models. While 8 out of these 12 binaries have clearly much higher initial system masses than those systems considered here, the remaining four (WR3, WR31, WR97, and WR139; cf. van der Hucht 2000) all have mass ratios of $M_{\mathrm{OB}} / M_{\mathrm{WR}} \lesssim 3$, and mostly much shorter periods than our models.

There are two possible ways to interpret the disagreement between our models and the observed $\mathrm{OB}+\mathrm{WN}$ binaries. One possibility is that our assumption of conservative evolution does not hold for systems with primaries of initially $\sim 25 M_{\odot}$. This can be motivated by the increasing role of radiation pressure in higher mass systems (cf., Schuerman 1972; Vanbeveren 1978). On the other hand, strong non-conservative effects in massive systems may result in an uncomfortably large upper critical ZAMS mass for neutron star formation of $\sim 40 M_{\odot}$, as imposed by the massive X-ray binary Wray 977 (Kaper et al. 1995; Ergma \& van den Heuvel 1998; Vanbeveren 1998b).

The other possibility involves to estimate the probability of finding one system of the kind computed here among four OB+WR binaries. As outlined in Sect. 5.1, the parameter range for conservative evolution becomes narrower for higher masses. For our systems with $25 M_{\odot}$ primaries, contact is avoided for an initial period of $4 \mathrm{~d}$, while $5 \mathrm{~d}$ resulted already in contact (cf. systems Nos. 65...74 in Table 3). Assuming initially an equal number of binaries per $\log P$-interval, and an upper period limit for interaction of $2000 \mathrm{~d}$, results in a ratio of contact-free versus contact surviving binary systems of $1 / 3.7$ if all Systems with initial mass ratios $q \lesssim 0.6$ merge. If only the systems with initial mass ratios $q \lesssim 0.6$ and initial periods $P \lesssim 200 \mathrm{~d}$ merge this ratio becomes 1:6.6. Because it is not well known how much systems really merge - also an unknown fraction of the large period contact systems might actually lead to a merger rather than to a $\mathrm{OB}+\mathrm{WR}$ binary - we assume that the ratio is between 1:4 and 1:7. Thus the probability of finding one suitable binary among four may be of the order of $1 / 2$ or lower. However, the survivors of the non-conservative contact evolution end up as shorter period systems with a mass and luminosity ratio closer to one ${ }^{3}$ than the contact-free systems, and are thus detected much easier.

In summary, the failure to detect counterparts of our more massive post-mass transfer systems is inconclusive. For our lower mass systems, i.e. such with initial primary masses of $12 M_{\odot}$ and $16 M_{\odot}$, the chance to detect counterparts may even be lower than for our higher mass systems (cf. Table 4). In fact, we do not know of any suitable OB star with a $1 \ldots 3.6 M_{\odot}$ helium star companion. The object which comes closest to our models may be $\Phi$ Persei, a $\sim 9 M_{\odot}$ B star with a $53000 \mathrm{~K}$ hot $1.1 M_{\odot}$ companion in a $127 \mathrm{~d}$ orbit (Gies et al. 1998). Its properties, and the fact that the B star is spinning rapidly, support actually the possibility of a close-to-conservative evolution at least in this system. A more puzzling system which may be relevant in the context is $\lambda$ Sco, a $\sim 10.5 M_{\odot} \mathrm{B}$ dwarf with a massive white dwarf companion in a $\sim 6 \mathrm{~d}$ orbit (Berghöfer et al. 2000). Waters et al. (1989) speculate that unseen companions in a number of Be stars may actually be helium stars, in accordance with Pols et al. (1991) and Portegies Zwart (1995).

The lack of $\mathrm{OB}+\mathrm{He}$ star systems which are more massive than $\Phi$ Per but less massive than OB+WR systems is, anyhow, very remarkable. I.e., if the evolution of

\footnotetext{
3 This fits well to the properties of the WR binaries WR31, WR97, and WR139, while WR3 has most likely not experienced any mass transfer.
} 
binaries with $12 \ldots 16 M_{\odot}$ primaries would, for a big fraction of the possible period range, proceed nonconservatively and without leading to mergers, we would expect short period $\mathrm{OB}+\mathrm{He}$ star systems which are easier detectable, with a smaller mass- and luminosity ratio compared to our models. Therefore, a non-detection of such systems may be easiest understood if the conservative evolution makes up for a large fraction of the non-merging interacting binaries in the considered mass range.

Whichever way it is, there are two kinds of observations hinting for a large number of $\mathrm{OB}+\mathrm{He}$ star binaries waiting to be discovered. One is the existence of massive $\mathrm{X}$-ray binaries, which are likely evolving from an $\mathrm{OB}+\mathrm{He}$ star stage (e.g., Moers \& van den Heuvel 1989). The second is the relatively large number of types Ib/Ic supernovae, which are mostly attributed to exploding helium stars with masses above $2 M_{\odot}$ (Podsiadlowski et al. 1992). I.e., it might be a rewarding project to attempt an observational search for helium star components to OB stars. In that respect, Table 4 can give indications of the expected parameters, with the remark that if mass transfer is partly non-conservative, then mass ratio, luminosity ratio, period and orbital velocities become more favourable for a detection.

Assuming a ratio of interacting binaries to single stars of 0.3 , and ignoring the unknown fraction of mergers among the binaries, implies that 1 out of $3 \mathrm{OB}$ stars in the considered mass range would, during its life time, obtain a He star companion. Depending of the rejuvenation of the secondary, this phase may last a considerable fraction, say one third, of the secondary's life time. This optimistic estimate indicates that only one out of ten OB stars in the considered mass range may have a helium star companion. However, these OB stars have a surface composition which is typically enriched in helium by $10 \ldots 20 \%$, and which has a N/C-ratio of about one order of magnitude above solar (cf. Sect. 3, and Wellstein \& Langer 2001). I.e., even though also single stars may become heliumand nitrogen-rich during their main sequence evolution due to rotational mixing (Heger \& Langer 2000), a preselection of chemically enriched OB stars may lead to a large detection probability of helium star companions.

Another way to pre-select OB stars with potential helium star companions is to identify OB type blue stragglers in young clusters or associations. The accretion of substantial amounts of matter by the primary may lead it to achieve a mass which is already above the turn-off mass of the cluster or associations. E.g., the $7.5 M_{\odot}$ Case A secondary shown in Fig. 1 grows to about $18 M_{\odot}$ at an age of $13 \mathrm{Myr}$, which is the turn-off age for $13 \ldots 14 M_{\odot}$. The $15 M_{\odot}$ Case B primary, upon turn-off of its $16 M_{\odot}$ companion, even grows to $27 M_{\odot}$. The remaining main sequence life time of the secondaries after accretion is of the order of $1 \mathrm{Myr}$, which may be long enough to catch them during this stage. Furthermore, the accretion of angular momentum may spin up the star, and lead to internal mixing processes and thereby to an overluminosity of the star with respect to its mass.
In summary, enriched surface abundances, blue straggler nature, overluminosity for the actual mass, and rapid rotation in an $\mathrm{O}$ or early B star may be hints to a helium star companion.

\section{Summary and conclusions}

In this paper, we have, on the basis of evolutionary models with up-to-date input physics, investigated the regime of contact-free evolution in close binaries with initial primary masses in the range $12 \ldots 25 M_{\odot}$, under the assumption that contact-free systems evolve conservatively, i.e., that no matter except stellar winds leaves these systems. Our results confirm earlier obtained ones qualitatively (e.g., Pols 1994), but differ quantitatively and extend over a wider parameter range.

We demonstrate that the number ratio of contact free Case A-to-Case B systems is a strong function of the system mass. At the high mass end $\left(25 M_{\odot}\right.$ initial primary mass), the contact-free Case B channel is closed completely, while contact-free Case A systems exist for a wide parameter range (cf. Table 3), although we find this range to depend strongly on the semiconvective mixing efficiency (cf. Sect. 5, and Wellstein \& Langer 1999). In contrast, the contact-free Case A channel becomes narrow at $12 M_{\odot}$ initial primary mass, whatever the assumptions on semiconvective mixing are, while the contact-free Case $B$ range becomes large (Fig. 12). At an initial primary mass of $16 M_{\odot}$, the situation is intermediate (Fig. 13).

We investigate the vastly different types of contact situations of systems in the considered mass range, and determine the critical periods and mass ratios to avoid contact (Sect. 5.1). We also estimate the consequences of the various kinds of contact. As a new phenomenon, we find that in what we call delayed contact, Case B systems can transfer the majority of the primary's envelope conservatively before - towards the end of the Case B mass transfer - contact and possibly a common envelope evolution occur. We find the delayed contact regime may lead, with increasing initial period, to a smooth transition from conservative contact-free evolution to the classical common envelope evolution, with the parameter $\beta$, which describes which fraction of the primary's envelope is accreted by the secondary, varying smoothly from $\beta=0$ to $\beta=1$. We are able to compute values of $\beta$ for our delayed contact systems, which we find in the range $\beta=0.4 \ldots 0.7$.

How far or close our model sequences actually are to the evolution of binary systems in nature depends to a large extent on the question whether contact-free systems do indeed evolve conservatively. I.e., the accreting star is likely to be rapidly spun-up to close to critical rotation (e.g., Packet 1981), and it depends on unknown viscous time scales whether or not it will be able to continue to grow in mass (Paczyński 1991). This question can in principle be answered by comparing our post-mass transfer models with corresponding observed binaries. However, as outlined in Sect. 6, even though virtually no observed counterparts to our post-mass transfer models - i.e., no 
$\mathrm{OB}+\mathrm{He}$ star systems with large mass ratios and orbital periods - are known, we could not conclude that conservative evolution in massive binaries is not realised in nature, for two reasons. First, identifying rather far and faint helium star companions to bright OB stars may be too difficult. Second, non-conservative evolution predicts even easier detectable $\mathrm{OB}+\mathrm{He}$ star systems but, again, no such systems are known, at least not corresponding to primary masses between 12 and $20 M_{\odot}-$ while three known $\mathrm{OB}+\mathrm{WN}$ binaries might indeed be the result of non-conservative binary evolution with primaries above $20 M_{\odot}$.

We want to emphasise that an answer to the question whether massive binaries can evolve conservatively is important to understand the late evolutionary stages: e.g., types Ib/Ic supernovae, massive X-ray and black hole binaries, and $\gamma$-ray bursts due to massive binaries. In principle one may constrain the conservativity of massive binaries by observations of the advanced stages. However, assumptions on even more uncertain processes have to be made in such attempts - e.g., on common envelope evolution, neutron star birth kicks, etc. - which strongly limits the success of such approaches. Furthermore, often, e.g. in population synthesis studies, only statistical information is obtained. For example, Dalton \& Sarazin (1995) find the period distribution of massive X-ray binaries consistent with an average loss of $\sim 70 \%$ of the transferred envelope mass. However, this can neither support nor defeat the existence of conservative evolution for contact-free massive binaries.

The very best way to constrain the first major mass transfer phase in massive close binaries would indeed be to identify a sample of helium star companions to OB stars. We repeat that those are predicted by conservative and by non-conservative binary evolution models, but with different periods and mass ratios. They are also indirectly proven to exist abundantly by the relatively large number of types Ib/Ic supernovae and massive X-ray binaries. In Sect. 6, we identify several ways to pre-select OB star candidates with an improved probability for a helium star companion.

Acknowledgements. We are grateful to Doug Gies, Lex Kaper, Jerry Orosz, and Philipp Podsiadlowski for many helpful discussions and suggestions and to Karel van der Hucht for providing us with results prior to publication. This work has been supported by the Deutsche Forschungsgemeinschaft through grants La 587/15 and La 587/16.

\section{References}

Benson, R. S. 1970, Ph.D. Thesis, Univ. of California, Berkeley Berghöfer, T., Vennes, S., \& Dupuis, J. 2000, ApJ, 538, 854

Braun, H. 1997, Ph.D. Thesis, LMU München

Braun, H., \& Langer, N. 1995, A\&A, 297, 483

Brookshaw, L., \& Tavani, M. 1993, ApJ, 410, 719

Chevalier, C, \& Ilovaisky, S. A. 1998, A\&A, 330, 201

Cox, A. N., \& Stewart, J. N. 1970, ApJS, 19, 261

Dalton, W. W., \& Sarazin, C. L. 1995, ApJ, 440. 280
Ergma, E., \& van den Heuvel, E. P. J. 1998, A\&A, 331, L29

Fliegner, J., Langer, N., \& Venn, K. A. 1996, A\&A, 308, L13

Fryer, C. L., Woosley, S. E., \& Hartmann, D. H. 1999, ApJ, 526,152

Gies, D. R., Bagnuolo, W. G., Ferrara, E. C., et al. 1998, ApJ, 493, 440

De Greve, J. P., \& de Loore, C. 1992, A\&ASS, 96, 653

Habets, G. M. H. J. 1986a, A\&A, 167, 61

Habets, G. M. H. J. 1986b, A\&A, 165, 95

Harries, T. J., \& Hilditch, R. W. 1997, MNRAS, 291, 544

Heger, A., \& Langer, N. 2000, ApJ, 544, 1016

Heger, A., Langer, N., \& Woosley, S. E. 2000, ApJ, 528, 368

Hellings, P. 1983, Ap\&SS, 96, 37

Hellings, P. 1984, Ap\&SS, 104, 83

Huang, R. Q., \& Taam, R. E. 1990, A\&A, 236, 197

van der Hucht, K. A. 2000, New Astron. Rev., in press

Iglesias, C. A., \& Rogers, F. J. 1996, ApJ, 464, 943

Kaper, L., Lamers, H. J. G. L. M., Ruymaekers, E., van den Heuvel, E. P. J., \& Zuiderwijk, E. J. 1995, A\&A, 300,446

Kippenhahn, R. 1969, A\&A, 3, 83

Kippenhahn, R., \& Thomas, H.-C. 1979, A\&A, 75, 281

Kippenhahn, R., Ruschenplatt, G., \& Thomas, H.-C. 1980, A\&A, 91, 175

Kolb, U., \& Ritter, H. 1990, A\&A, 236, 385

Kopal, Z. 1978, Dynamics of Close Binary Systems (D. Reidel, Dordrecht)

Langer, N. 1991, A\&A, 252, 669

Langer, N. 1992, A\&A, 265, L17

Langer, N. 1998, A\&A, 329, 551

Langer, N., Fricke, K. J., \& Sugimoto, D. 1983, A\&A, 126, 207

de Loore, C., \& De Greve, J. P. 1992, A\&ASS, 94, 453

de Loore, C., \& Vanbeveren, D. 1992, A\&A, 260, 273

Maeder, A. 1987, A\&A, 178, 159

Martin, C., Mignard, F., Hartkopf, W. I., \& McAlister, H. A. 1998, A\&AS, 133, 149

Meyer, F., \& Meyer-Hofmeister, E. 1983, A\&A, 121, 29

Mochnacki, S. W. 1984, A\&AS, 55, 551

Moers, E. J. A., \& van den Heuvel, E. P. J. 1989, A\&A, 226, 88

Neo, N., Shigeki, M., Nomoto, K., \& Sugimoto, D. 1977, PASJ, 29, 249

Ostrov, P. G., Lapasset, E., \& Morrell, N. I. 2000, A\&A, 356, 935

Packet, W. 1981, A\&A, 102, 17

Paczyński, B. 1967, Acta Astron., 17, 193

Paczyński, B. 1991, ApJ, 370, 597

Paczyński, B., \& Sienkiewicz, R. 1972, Acta Astron., 22, 73

Pastetter, L., \& Ritter, H. 1989, A\&A, 214, 186

Plavec, M., Ulrich, R. K., \& Polidan, R. S. 1973, PASP, 85, 769

Podsiadlowski, Ph. 1992, PASP, 104, 717

Podsiadlowski, Ph., Joss, P. C., \& Rappaport, S. 1990, A\&A, 227, L9

Podsiadlowski, Ph., Joss, P. C., \& Hsu, J. J. L. 1992, ApJ, 391, 246

Pols, O. R. 1994, A\&A, 290, 119

Pols, O. R., Cote, J., Waters, L. B. F. M., \& Heise, J. 1991, A\&A, 241, 419

Portegies Zwart, S. F. 1995, A\&A, 296, 691

Press, W. H., Flannery, B. P., Teukelsky, S. A., \& Vetterling W. T. 1988, Numerical Recipes in C (Cambridge University Press) 
Ritter, H. 1988, A\&A, 202, 93

Savonije, G. J. 1978, A\&A, 62, 317

Savonije, G. J., \& Papaloizou, J. C. B. 1997, MNRAS, 291, 633

Schuerman, D. W. 1972, Ap. Space Sci., 19, 351

Shigeyama, T., Nomoto, K., Tsujimoto, T., \& Hashimoto, M. 1990, ApJL, 361, L23

Stern, M. E. 1960, Tellus, 12, 172

Sybesma, C. H. B. 1985, A\&A, 142, 171

Ulrich, R. K. 1972, ApJ, 172, 165

Ulrich, R. K., \& Burger, H. L. 1976, ApJ, 206, 509

Vanbeveren, D. 1978, Ap. Space Sci., 57, 41

Vanbeveren, D., Van Rensbergen, W., \& De Loore, C. 1998a,

The Brightest Binaries (Kluwer Academic Publishers, Dordrecht)
Vanbeveren, D., De Loore, C., \& Van Rensbergen, W. 1998b, A\&AR, 9, 63

Vanbeveren, D., De Donder, E., Van Bever, J., Van Rensbergen, W., \& De Loore, C. 1998c, New Astron., 3, 443

Waters, L. B. F. M., Pols, O. R., Hogeveen, S. J., Cote, J., \& van den Heuvel, E. P. J. 1989, A\&A, 220, L1

Webbink, R. F. 1984, ApJ, 277, 355

Wellstein, S., \& Langer, N. 1999, A\&A, 350, 148

Wellstein, S., \& Langer, N. 2001, in preparation

Woosley, S. E., Langer, N., \& Weaver, T. A. 1995, ApJ, 448, 315 